\title{
Entrainment and motion of coarse particles in a shallow water stream down a steep slope
}

\author{
C. ANCEY ${ }^{1}$, A. C. DAVISON ${ }^{2}$, T. BÖHM$M^{3}$, M. JODEAU \\ AND P. FRE Y P $^{3}$ \\ ${ }^{1}$ School of Architecture, Civil and Environmental Engineering, École Polytechnique Fédérale de \\ Lausanne, 1015 Lausanne, Switzerland \\ ${ }^{2}$ Institute of Mathematics, École Polytechnique Fédérale de Lausanne, 1015 Lausanne, Switzerland \\ ${ }^{3}$ Cemagref, Domaine Universitaire BP 76, 38402 Saint-Martin-d'Hères Cedex, France \\ ${ }^{4}$ Cemagref, 3 bis quai Chauveau, 69336 Lyon, France
}

(Received 28 October 2006 and in revised form 21 August 2007)

We investigate the entrainment, deposition and motion of coarse spherical particles within a turbulent shallow water stream down a steep slope. This is an idealization of bed-load transport in mountain streams. Earlier investigations have described this kind of sediment transport using empirical correlations or concepts borrowed from continuum mechanics. The intermittent character of particle transport at low-water discharges led us to consider it as a random process. Sediment transport in this regime results from the imbalance between entrainment and deposition of particles rather than from momentum balance between water and particles. We develop a birth-death immigration-emigration Markov process to describe the particle exchanges between the bed and the water stream. A key feature of the model is its long autocorrelation times and wide, frequent fluctuations in the solid discharge, a phenomenon never previously explained despite its ubiquity in both nature and laboratory experiments. We present experimental data obtained using a nearly two-dimensional channel and glass beads as a substitute for sediment. Entrainment, trajectories, and deposition were monitored using a high-speed digital camera. The empirical probability distributions of the solid discharge and deposition frequency were properly described by the theoretical model. Experiments confirmed the existence of wide and frequent fluctuations of the solid discharge, and revealed the existence of long autocorrelation time, but theory overestimates the autocorrelation times by a factor of around three. Particle velocity was weakly dependent on the fluid velocity contrary to the predictions of the theoretical model, which performs well when a single particle is moving. For our experiments, the dependence of the solid discharge on the fluid velocity is entirely controlled by the number of moving particles rather than by their velocity. We also noted significant changes in the behaviour of particle transport when the bed slope or the water discharge was increased. The more vigorous the stream was, the more continuous the solid discharge became. Moreover, although $90 \%$ of the energy supplied by gravity to the stream is dissipated by turbulence for slopes lower than $10 \%$, particles dissipate more and more energy when the bed slope is increased, but surprisingly, the dissipation rate is nearly independent of fluid velocity. A movie is available with the online version of the paper. 


\section{Introduction}

A longstanding problem in the study of bed load transport in gravel-bed rivers and mountain streams is related to the physical mechanisms governing bed resistance and particle motion. There remains a wide gap between field measurements and the predictions of theoretical models, despite their capacity to describe bed load transport for laboratory experiments correctly. For instance, the sediment flow rates measured in gravel-bed rivers may differ within one to two orders of magnitude from the solid discharges predicted by bed-load transport equations (Gomez \& Church 1989; Wilcock 2001; Martin 2003; Barry, Buffington \& King 2004).

Sediment transport has been attacked in many ways over the last century. The historical approach consisted in seeking empirical relations between the solid discharge and the water flow rate by correlating data obtained from flume experiments. For instance, Meyer-Peter \& Müller (1948) used a large amount of laboratory data to derive an equation that provides the solid discharge as a function of the water flow rate, bed gradient and particle size distribution. This equation, which was revisited and extended (Smart \& Jaeggi 1983; Rickenmann 2001; Wong \& Parker 2006), is often used in engineering applications and research. There have also been many attempts to develop analytical models from physical considerations. Of the numerous mechanistic descriptions of bed load transport (for a review, see Graf 1984; Raudkivi 1990), two main approaches have emerged: Bagnold's and Einstein's formulations (Seminara, Solari \& Parker 2002).

In Bagnold's approach, the solid discharge equation can be derived by considering the balance between the energy supplied by gravity and that expended by turbulence and sediment transport (Bagnold 1966, 1973). In Bagnold's model and subsequent variants (Wiberg \& Smith 1989; van Rijn 1985; Niño \& Garcia 1998), bed load transport is essentially a two-phase flow whose dynamics are controlled by the momentum transfers between the water and solid phases. When compared with laboratory experimental data or field measurements, Bagnold's scaling correctly describes the sediment transport for steady uniform or gently varying flows at sufficiently high solid discharges (Julien 1994). However, there are several indications that Bagnold's formulation may be flawed or crude. First, for this scaling to match experimental observations, the bulk particle friction coefficient introduced by Bagnold must be fitted to non-physical values (Fernandez Luque \& van Beek 1976; Niño, Garcì \& Ayala 1994). Secondly, for conditions that depart from steady uniform flow, Bagnold's model yields poor results, notably for flows over arbitrarily sloping beds (Seminara et al. 2002) or at low levels of solid discharge (Fernandez Luque \& van Beek 1976; Nelson et al. 1995). Thirdly, if bedforms (dune or antidune, depending on the value of the Froude number) are interpreted as resulting from a loss of linear stability in the coupled fluid-solid system, Bagnold's model fails to capture the necessary physics, since the corresponding equations of motion show no instability (Balmforth \& Provenzale 2001).

In Einstein's view, sediment transport does not result from an equilibrium in the momentum transfers between solid and liquid phases, but rather from the difference between the entrainment and deposition rates, $E$ and $D$, respectively, which are a function of the flow conditions and bed geometry (Einstein 1950; Ettema \& Mutel 2004). This amounts to writing that on a small interval $\Delta x$, the solid discharge variation is $\delta q_{s}=(E-D) \Delta x$, and so the solid discharge at bed equilibrium is the implicit solution to the equation $E=D$. Einstein's stochastic approach poses several problems that have as yet few solutions. For instance, as particles move sporadically and in different groups, the solid flow rate comprises a series of pulses and is highly 
fluctuating, making it difficult to define and measure properly, even under steady flow conditions (Bunte \& Abt 2005). Another problem is the proper definition of the characteristic time of entrainment and deposition rates, controversy about which has led to several attempts to refine Einstein's original formulation (Paintal 1971; Laursen 1999; Lopez \& Garcia 2001; Kleinhans \& van Rijn 2002; Charru, Mouilleron \& Eiff 2004; Cheng 2004; Cheng, Tang \& Zhu 2004). Other aspects, such as the abnormal diffusion of bed particles or wide fluctuations in the solid discharge, seriously conflict with the predictions of Einstein-like theories (Nikora et al. 2001, 2002; Ancey et al. 2006)-both field and laboratory experiments have revealed that the instantaneous solid discharge is frequently three to four times higher than its mean value (Kuhnle \& Southard 1988; Lisle 1989; Böhm et al. 2004), suggesting that the probability density functions of the transport-rate records have a thick tail and thus depart from the expected Gaussian behaviour. This can be seen as the hallmark of collective motions (Sornette 2000); if so, it also undermines any mean-field approximation in which cooperation between particles is unaccounted for.

Both approaches provide solid discharge equations that agree quite well with laboratory flume experiments and, to some degree, with field measurements in rivers, but both require the use of fitting procedures to estimate empirical parameters. A full analytical approach seems intractable owing to the many processes involved: complex interplay between the particles and the carrying fluid; particle exchanges between the bed and the flow; turbulence effects (bed friction, advection of turbulent structures); and so forth. As in many other problems in the environmental sciences, it is difficult to judge the reliability of a model some of whose parameters must be tuned (Iverson 2003), as the judgement may be biased owing to the parameter fitting. Rather than immediate applicability, progress requires an enhanced understanding of physical processes and the testing of models by assessing the range over which the discrepancies with measurements are minor. Moreover, if we focus on solving all the specific problems of turbulent flow in particle systems, we may lose sight of the original objective, of finding a simple but general analytical framework for dealing with sediment-transport issues in practical situations.

The present paper is a step towards such a framework, made by thoroughly examining physical processes involved in sediment transport, while limiting the complexity of our description. A key factor was access to detailed data on the flows and measurements on as many parameters as possible. We avoided complications associated with many laboratory experiments when working with natural sediment by devising a simple system in which the boundary and initial conditions can be entirely controlled and imposed. We built a narrow channel, in which sediment was replaced by coarse spherical glass beads. As our aim was not to provide a comprehensive picture of sediment transport, we focused on steep slopes and coarse particles, for which transport of particles is referred to as bedload transport-particles are not in suspension, but roll or jump along the bed. The resulting flow conditions are typical of those encountered in steep gravel-bed rivers in piedmont and mountain regions, but are not representative of thick flows on shallow slopes.

Section 2 outlines our theoretical framework. We wish to compute the solid discharge, which can be defined as the product of the number of moving particles and the mean particle velocity. In any theory of sediment transport, the crux lies in the proper evaluation of the number of particles entrained and maintained in motion by the water stream. In Bagnold's approach, sediment transport results from an equilibrium in the momentum transfer between solid and liquid phases. The number of moving particles is then found to be the ratio between the available energy 
and that expended during the motion of one particle due to momentum transfers between the solid and liquid phases. The mean-field treatment applied by Bagnold conflicts with the observed intermittent nature of sediment transport at low flow rates. Both laboratory and field measurements reveal that particle movement occurs for a period of time followed by a period in which no motion occurs (Drake et al. 1988; Niño \& Garcì 1998). At low flow rates, intermittent motion occurs when the near-bed downstream velocity is sufficiently high to destabilize stationary particles and set them in motion, corresponding to instances in which the instantaneous near-bed velocity outweighs its mean value as a result of near-bed turbulence and does not correlate well with the mean flow conditions far from the bed (Nelson et al. 1995, 2001; Schmeeckle \& Nelson 2003). In Einstein's theory, sediment transport results from the imbalance between entrainment and deposition. Intermittency in particle motion is usually taken into account by treating it as a two-state Markov process (Lisle et al. 1998; Papanicolaou et al. 2002; Ancey et al. 2006). A severe shortcoming of existing models is that they give unrealistic solid discharge fluctuation distributions; for instance, they predict that the solid discharge gently fluctuates around its mean value for equilibrium flow conditions, whereas experiments reveal the existence of wide and frequent fluctuations (Ancey et al. 2006). Here, we develop a generalized birth-death process to describe the time variation in the number of moving particles. Our simple stochastic model overcomes the current limitations of Einstein-like models and predicts a number of interesting features, such as the autocorrelation time of the solid discharge.

In $\S 3$, we describe our experimental facility. We run experiments in an inclined tight flume with a continuous particle supply and steady flow rate. This nearly twodimensional flume is assumed to be the simplest representation of sediment transport on the laboratory scale and presents a number of advantages: the boundary conditions can be controlled and most of the flow variables can be measured by image processing. Section 4 is devoted to experimental results and comparison with our theoretical model. Since a quantitative comparison between theory and experiment is biased by any parameter fitting, we test theory by analysing the probabilistic features of the model. In $\S 5$, we summarize our findings and look ahead a little.

\section{Theoretical framework}

\subsection{Objective and notation}

We consider a two-dimensional steady water stream flowing down a bed of mean slope $\theta$, and comprising mobile spherical particles of equal radius $a$ and density $\varrho_{p}$. The water flow rate per unit width $q_{w}$ is prescribed at the channel entrance, and the flume is supplied with particles identical to those in the bed. Figure 1 shows the flow configuration.

A few solid particles are entrained by the water stream: they can roll/slide along the bed or they can leap and stay in saltation in the water stream for short time periods. We refer to the former motion as the rolling regime and to the latter as the saltating regime. As we are especially interested in weakly intense bed-load transport, we stress flows with a fairly low fluid velocity: the trajectory of a single particle then exhibits a succession of rests and moves in a rolling or saltating regime, making it difficult to discriminate the motion regime accurately. Below, we do not discriminate between rolling and saltation, except when stated explicitly, and treat both motions as a single species which we call the moving particles. 


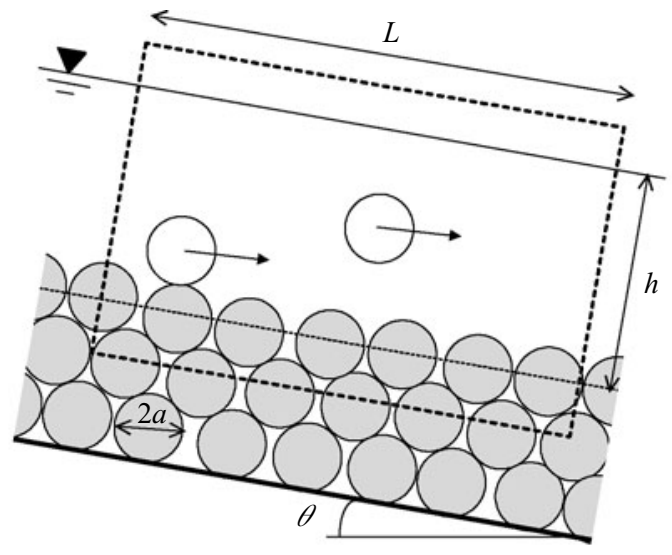

FIGURE 1. Definition of the flow configuration.

In a typical bed-load transport problem, we wish to determine properties of the flow such as its depth $h$, the solid discharge (the volume of particles per unit time conveyed by the flow) $q_{s}$ and the mean flow $\bar{u}_{f}=q_{w} / h$, as functions of the flow configuration parameters $\left(q_{w}, \theta, a, \varrho_{p}\right)$. We consider a control volume $\mathscr{V}$ within which we follow the particles and the motion of the fluid; the volume $V=\mathscr{S} \times L$ of $\mathscr{V}$ is a function of its length $L$ and its cross-section $\mathscr{S}=h W$, where $W$ represents the flume width.

In this section, we restrict our attention to sediment transport at equilibrium, i.e. on average, flow is steady and there is neither deposition nor erosion along the bed. In the next subsection we show that the solid discharge can be computed as $q_{s} \propto N \bar{u}_{p}$, where $N$ denotes the number of particles within the control volume $\mathscr{V}$ and $\bar{u}_{p}$ is the mean particle velocity. In $\S 2.2$, we develop a stochastic model for the number of particles in $\mathscr{V}$, while $\S 2.5$ is devoted to particle velocity. In $\S 2.2$, we derive a partial differential equation governing the time variations in the probability $P(n ; t)$ of finding $N=n$ moving particles in the control volume at time $t$. General and stationary solutions to the master equation are derived in $\S 2.3$ and $\S 2.4$, respectively.

\subsection{Birth-death emigration-immigration process}

The solid discharge can be defined as the flux of particles through a flow crosssection $\mathscr{S}: q_{s}=\int_{\mathscr{S}} \boldsymbol{u}_{p} \cdot \boldsymbol{k} \mathrm{d} \mathscr{S}$, where $\boldsymbol{k}$ is the unit normal to $\mathscr{S}$. This definition, suitable for continuous fields, is not well suited to discrete elements, for which it is more convenient to introduce the flow rate in terms of the probability $P\left[\boldsymbol{u}_{p} \mid \boldsymbol{x}, t\right]$ that a particle crosses the control surface $\mathscr{S}$ at position $\boldsymbol{x}$ and time $t$ with velocity $\boldsymbol{u}_{p}$,

$$
q_{s}=\int_{\mathscr{S}} \int_{\mathbb{R}^{2}} P\left[\boldsymbol{u}_{p} \mid \boldsymbol{x}, t\right] \boldsymbol{u}_{p} \cdot \boldsymbol{k}|\mathrm{d} \boldsymbol{x}| \mathrm{d} \boldsymbol{u}_{p} .
$$

Under steady conditions we have $\partial P / \partial t=0$ and so this definition reduces to

$$
q_{s}=\lim _{V \rightarrow \infty} \frac{1}{V} \sum_{i=1}^{N} u_{i} v_{p} \mathscr{S}=\lim _{L \rightarrow \infty} \frac{v_{p}}{L} \sum_{i=1}^{N} u_{i},
$$

where the ensemble average is replaced by a volume average and $v_{p}$ is the particle volume. Integration has been performed over the control volume $\mathscr{V}$. We let $u_{i}=\boldsymbol{u}_{p} \cdot \boldsymbol{k}$ denote the streamwise velocity component of particle $i$. Below we define the flow rate 


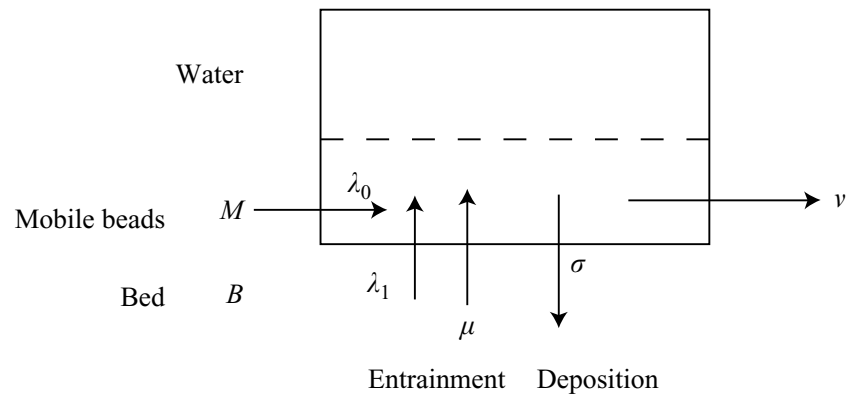

FIGURE 2. The number of moving beads observed within the window varies with time, depending on the number of particles entering/leaving the window or being entrained/deposited from/ on the stationary bed.

$\dot{n}=q_{s} / v_{p}$ as

$$
\dot{n}=\frac{1}{L} \sum_{i=1}^{N} u_{i} .
$$

In order to compute the discharge equation, we must establish the number $N$ of particles in motion and their velocities, which depend on $q_{w}, \theta, a$ and $\varrho_{p}$. We assume that the number of stationary beads in the bed is infinite.

We now consider the total number $N(t)$ of particles in motion within the control volume $\mathscr{V}$ at time $t$. Figure 2 sketches the particle exchanges within the control volume. We describe sediment transport using an immigration-emigration birth-death process (Cox \& Miller 1965), using the following exchanges.

(i) A moving bead enters the window at rate $\lambda_{0}>0$ by rolling/saltating from the left (immigration). The corresponding probability of arrival of a particle in the time interval $[t, t+\Delta t)$ is independent of $t$ and $N$, and we can write

$$
P(n \rightarrow n+1 ; \Delta t)=\lambda_{0} \Delta t+o(\Delta t) .
$$

(ii) Moving beads leave the window independently at rate $v>0$ (emigration). The transition probability is

$$
P(n \rightarrow n-1 ; \Delta t)=n v \Delta t+o(\Delta t) .
$$

(iii) Two processes enable entrainment of particles from the bed (birth): a particle can be dislodged from the bed by the water stream at rate $\lambda_{1}>0$; or a moving bead can destabilize a stationary one and set it moving. This occurs at rate $\mu$ for any moving bead within the observation window. The corresponding transition probabilities are, respectively,

$$
P(n \rightarrow n+1 ; \Delta t)=\lambda_{1} \Delta t+o(\Delta t), \quad P(n \rightarrow n+1 ; \Delta t)=\mu n \Delta t+o(\Delta t) .
$$

(iv) A moving bead can settle (i.e. come to rest) within the window, independently at rate $\sigma$ for each moving bead (death). The transition probability is thus

$$
P(n \rightarrow n-1 ; \Delta t)=n \sigma \Delta t+o(\Delta t) .
$$

With these assumptions and using the usual arguments for setting up the forward equations of a Markov process with discrete states in continuous time (Cox \& Miller 1965; Gardiner 1983), we obtain a set of equations

$$
\begin{aligned}
P(n ; t+\Delta t)= & \alpha(n+1) \Delta t P(n+1 ; t)+P(n-1 ; t)\{\lambda+(n-1) \mu\} \Delta t \\
& +P(n ; t)\{1-\Delta t(\lambda+n \alpha+n \mu)\}+o(\Delta t),
\end{aligned}
$$


for $n=1,2, \ldots$, and

$$
P(0 ; t+\Delta t)=\alpha P(1 ; t) \Delta t+P(0 ; t)(1-\lambda \Delta t)+o(\Delta t),
$$

for $n=0$, with the shorthand notation $\alpha=\sigma+v$ and $\lambda=\lambda_{1}+\lambda_{0}$. The time increment $\Delta t$ is assumed to be sufficiently small that two events cannot occur in $(t, t+\Delta t)$. On rearranging the terms and letting $\Delta t \rightarrow 0$, we obtain

$$
\begin{gathered}
\frac{\partial}{\partial t} P(n ; t)=(n+1) \alpha P(n+1 ; t)+(\lambda+(n-1) \mu) P(n-1 ; t)-(\lambda+n(\alpha+\mu)) P(n ; t), \\
\frac{\partial P(0, t)}{\partial t}=\alpha P(1 ; t)-\lambda P(0 ; t)
\end{gathered}
$$

At time $t=0$, there are $N=N_{0}$ particles within the control volume, so we set

$$
P(n ; 0)=\delta\left(n-N_{0}\right),
$$

where $\delta$ is the Kronecker delta function.

We now discuss the main assumptions underlying (2.8). The key aspects of our model are the entrainment processes and the physical origin of the mathematical formulation, its limitations, and the nature of the parameters $\lambda_{0}, \lambda_{1}, \mu, v$ and $\sigma$.

The main originality of the model lies in the treatment of particle entrainment. In Einstein-like theories, particles are entrained when the drag/lift force exerted by the water stream exceeds the resisting force (weight and bed friction) of a stationary particle (see below), and there is no collective entrainment $(\mu=0)$. This results in a purely Poissonian representation of entrainment (Lisle et al. 1998; Papanicolaou et al. 2002; Ancey et al. 2006), which reflects the erosive action of water only and is independent of the number of particles previously entrained. It implies that the total number of moving particles varies little with time, in conflict with experimental and field observations (Ancey et al. 2006). Our model includes an additional entrainment process that depends on the number of moving particles, and reflects the destabilization of bed particles and their entrainment owing to interactions with moving particles. These interactions include direct collisions and softer interactions. The former process was clearly identified in experiments and field surveys (Drake et al. 1988; Böhm et al. 2004). In this case, the effect is close to the splash function used for modelling sand drift, although the physical processes are quite different; in particular, momentum exchanges during a collision are rapidly dampened by viscous forces in water, which explains why particle ejection is not usually observed in water (Schmeeckle et al. 2001). The latter process refers to the significant modifications in the velocity profile when a moving particle approaches a stationary bead, which may increase the force exerted by the fluid on this stationary particle. Since both effects reflect interactions with moving particles, resulting entrainment is called collective entrainment. Such entrainment allows the probability distribution function of $N$ to accommodate the wide fluctuations seen in experiments.

In the model above, the total entrainment rate is $E=\lambda_{1}+\mu N$, while the deposition rate is $D=\sigma N$. Statistically, these equations cannot be symmetric because on the one hand, the number of stationary beads is assumed to be infinite and, on the other hand, the number of moving particles is finite. For entrainment, we consider that a particle can spontaneously start moving as a result of water action or be entrained because of the disturbances induced by moving particles; in this case, these two distinct physical mechanisms give rise to two statistically different contributions, $\lambda_{1}$ and $\mu N$. For deposition, we can also imagine that a particle settles independently of other particles 
(a)

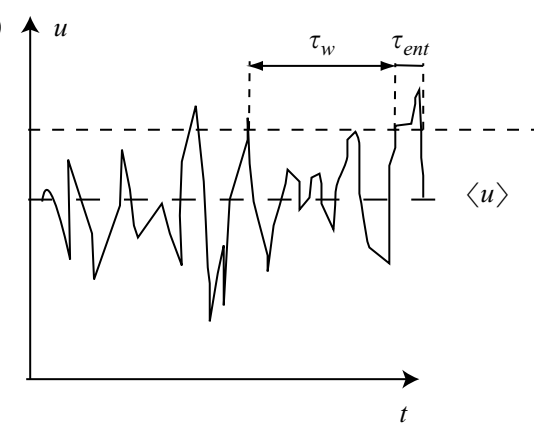

(b)

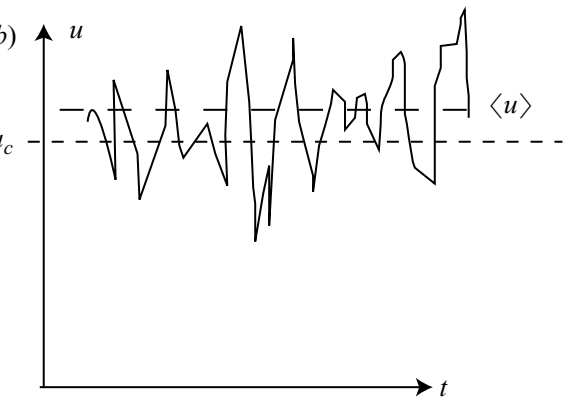

FIGURE 3. Fluctuations of the instantaneous velocity $u$ around the mean value $\langle u\rangle$. $(a)$ Mean fluid velocity below the critical threshold $u_{c}$. $(b)$ Mean fluid velocity in excess of the critical threshold $u_{c}$. In both cases, particle entrainment occurs sporadically.

or because its motion is impeded by bed particles; in that case, although the physical mechanics are different, the resulting probabilities have the same form, with a linear dependence on $n$. Following a parsimony principle, we do not discriminate between the physical processes governing deposition since they lead to statistical equations of the same form, whose parameters cannot be determined separately.

Our approach is likely to perform well for a limited range of water flow rates, which must be sufficiently high for the probability of entrainment to be appreciable, but not so high that entrainment occurs continuously. This can be more easily understood through figure 3, which represents the time variations in the instantaneous fluid velocity $u$ undergone by a bed particle under steady flow conditions (see $\S 2.5$ ). The mean fluid velocity is denoted by $\langle u\rangle(y)$, where $y$ is the height from a reference level and the angle brackets indicate time-averaged values. The force exerted on the particle by the fluid is usually split into hydrostatic pressure, drag and lift. In current models of entrainment on steep slopes, a particle starts to move whenever the combined action of gravity and fluid forces exceeds the particle weight and the frictional force (Kovacs \& Parker 1994; Armanini \& Gregoretti 2005); in these models, the mean lift and drag forces are directly linked to the mean fluid velocity $\langle u\rangle(y)$, so loss of stability and incipient motion occur whenever the instantaneous fluid velocity exceeds a critical threshold $u_{c}$, that depends on bed slope, particle arrangement, and so forth (Kirchner et al. 1990; Armanini \& Gregoretti 2005). The probability of entrainment is thus the exceedance probability $P\left(u>u_{c}\right)$ (Einstein 1950; Papanicolaou et al. 2002).

To determine how frequently these events occur, we require the characteristic time between two events. We refer to $\tau_{w}$ as the waiting time between events, and the mean duration of an entrainment event, during which $P\left(u>u_{c}\right)$, is denoted by $\tau_{\text {ent }}$ (see figure $3 a)$. The exceedance probability $P_{c}=P\left(u>u_{c}\right)$ gives the time ratio $\tau_{e} /\left(\tau_{w}+\tau_{e}\right)$. We introduce the Taylor macro scales of length and time, $L_{x}$, and of time, $\tau_{\text {turb }}$, which represent the correlation length and time of large turbulent structures, respectively. For open channel flows down rough surfaces, semi-empirical models give estimates of the large-eddy length scale in the form $L_{x}=A h \sqrt{y / h}$, where $A$ is a constant close to unity (Nezu \& Nakagawa 1993); for steady flow conditions, the autocorrelation time macro scale is then $\tau_{\text {turb }}=L_{x} /\langle u\rangle$. If the autocorrelation time function is close to exponential, and using the relation $1 / \tau_{\text {turb }}=1 / \tau_{w}+1 / \tau_{\text {ent }}$, then the waiting time is

$$
\tau_{w}=\frac{1-P_{c}^{2}}{P_{c}} \frac{L_{x}}{\langle u\rangle},
$$

thus the entrainment rate $\lambda_{1} \propto \tau_{w}^{-1}$ varies with the fluid velocity $\langle u\rangle$. 
This statistical description is likely to hold at intermediate bottom shear stress, i.e. for $\langle u\rangle<u_{c}$ or $\langle u\rangle>u_{c}$ (see figure 3) provided that $\left|\langle u\rangle-u_{c}\right|<u^{\prime}$, where $u^{\prime}$ denotes the root mean square velocity in the streamwise direction. Indeed, at very low shear stress, i.e. when $\langle u\rangle$ is far below the critical threshold, entrainment may occur on rare occasions when turbulent eddies that are on the same scale as the particle produce imbalanced pressure fields around the particle, leading to a high lift event; such events are strongly dependent on the details of the flow around of the particle and particle arrangement of the bed, which cannot be conveniently related to the average conditions (Nelson et al. 2001). Extreme value theory suggests that the number of instances in which a particle is lifted up from the bed will follow a Poisson distribution, but with a rate $\lambda_{1}$ independent of the mean velocity. Conversely, at high bottom shear stress (i.e. for $\langle u\rangle \gg u_{c}$ ), any bed particle exposed to the stream is instantaneously set in motion. In that case, bed load transport takes the form of a sheet flow separating the bed from the water flow (Jenkins \& Hanes 1998). This continuous process cannot readily be described within our stochastic framework.

\subsection{General solution}

We obtain the general solution to the forward master equations (2.8)-(2.9) subject to the initial condition $(2.10)$ by introducing the probability generating function (Gardiner 1983)

$$
G(z, t)=\sum_{n=0}^{\infty} z^{n} P(n ; t),
$$

which makes it possible to transform the master equations into a single partial differential equation. Multiplying (2.8)-(2.9) by $z^{n}$ and summing over $n$, we obtain

$$
\frac{\partial}{\partial t} G(z, t)=\lambda(z-1) G(z, t)+\left\{\sigma+\mu z^{2}+v-(\mu+\sigma+v) z\right\} \frac{\partial}{\partial z} G(z, t),
$$

whose general solution is

$$
G(z, t)=(\alpha-z \mu)^{-\lambda / \mu} h\left(\mathrm{e}^{-t(\alpha-\mu)} \frac{1-z}{\alpha-z \mu}\right)
$$

since $z<1$ and provided that $\alpha>\mu$ (see $\S 2.4$ ) so that a stationary solution exists. The function $h$ in (2.13) may be determined by the initial condition. If there are $n=N_{0}$ beads within the control volume at time $t=0$, then (2.13) yields

$$
G(z, 0)=z^{n}=(\alpha-z \mu)^{-\lambda / \mu} h\left(\frac{1-z}{\alpha-z \mu}\right),
$$

from which we obtain

$$
h(x)=\left(\frac{1-\alpha x}{1-\mu x}\right)^{n}\left(\frac{\alpha-\mu}{1-\mu x}\right)^{\lambda / \mu} .
$$

Thus the complete form of $G$, given that the initial state is known, is

$$
G(z, t)=\left(\frac{\alpha-\mu}{(K \mu-\mu) z+\alpha-K \mu}\right)^{n+\lambda / \mu}\left(\frac{(K \alpha-\mu) z+\alpha(1-K)}{\alpha-\mu}\right)^{n},
$$

where $K=\mathrm{e}^{-t(\alpha-\mu)}$ corresponds to the autocorrelation function for flows at equilibrium (see $\S 2.4$ ); note that $G(1, t)=1$ and $G(z, 0)=z^{n}$. The coefficient of $z^{m}$ in the power 
series expansion of (2.14) is the transition probability $\operatorname{prob}(N(t)=m \mid N(0)=n)$, which may be used for likelihood estimation of the parameters $\alpha, \lambda$ and $\mu$ when the system is out of equilibrium, as is often the case for laboratory and field measurements.

When $\mu=0, G$ is the probability generating function of the binomial distribution $\operatorname{Bin}(n, K)$, and we retrieve the behaviour expected under Einstein's theory (Ancey et al. 2006): the sporadic motion of each particle is described as a Bernoulli process, and since the sum of $N$ Bernoulli processes is a binomial process, $N$ is a binomial variable.

\subsection{Steady-state solution}

For steady flow conditions, the number of particles within the observation window forms a stationary random process, whose probability distribution $P_{s}(n)=\operatorname{Prob}(N=n)$ may be obtained by letting $t \rightarrow \infty$ in (2.14), thus yielding $G_{s}(z)$. For steady-state conditions, $G$ is independent of $t$ and we express it as $G_{s}(z)$; this turns out to be the probability generating function of the negative binomial distribution

$$
P_{s}(n)=\operatorname{NegBin}(n ; r, p)=\frac{\Gamma(r+n)}{\Gamma(r) n !} p^{r}(1-p)^{n}, n=0,1, \ldots,
$$

with $r=\lambda / \mu$ and $p=1-\mu / \alpha$, and where $\Gamma$ denotes the gamma function (Abramowitz $\&$ Stegun 1964). The mean is $\lambda /(\alpha-\mu)$ and the variance is

$$
\operatorname{Var} N=\frac{\lambda \alpha}{(\alpha-\mu)^{2}} .
$$

For $\mu=0$, we obtain $G_{s}(z)=\mathrm{e}^{-\lambda(z-1) / \alpha}$, corresponding to the Poisson distribution of rate $r^{\prime}=\lambda / \alpha$,

$$
P_{s}(n)=\frac{\left(r^{\prime}\right)^{n}}{n !} \mathrm{e}^{-r^{\prime}}, \quad n=0,1, \ldots
$$

A stationary distribution exists only if $\alpha>\mu$ : that is, the rate at which beads disappear is lower than their appearance rate. For steady flow conditions, there are additional constraints on the parameters $v, \mu, \sigma, \lambda_{0}$ and $\lambda_{1}$. On average, the number of particles that leave the observation window must match the number of particles that enter it,

$$
\mathbb{E}(N) \sigma+\mathbb{E}(N) v=\lambda_{0}+\lambda_{1}+\mathbb{E}(N) \mu,
$$

where $\mathbb{E}(N)$ is the time-averaged number of particles. Moreover, bed equilibrium implies that on average, there is no variation in the bed elevation over time,

$$
\mathbb{E}(N) \sigma=\lambda_{1}+\mathbb{E}(N) \mu,
$$

and that $\mathbb{E}(N) v=\lambda_{0}$ : the inflow matches the outflow.

Under stationary conditions, the autocorrelation function of the number of particles in motion within the window may be written as (Gardiner 1983)

$$
\operatorname{corr}\{N(0), N(\tau)\}=\rho(\tau)=\frac{\mathbb{E}[N(\tau) N(0)]-\mathbb{E}[N(0)]^{2}}{\operatorname{Var}\left(N_{0}\right)}, \quad \tau>0 .
$$

The mean of $N(\tau) N(0)$ can be expressed as $\mathbb{E}[N(0) \mathbb{E}\{N(\tau)\} \mid N(0)]$, in which the conditional mean of $N(\tau)$ given $N(0)$ appears; this can be obtained from equation (2.14) (Gardiner 1983),

$$
\mathbb{E}\{N(\tau) \mid N(0)=n\}=\left(\frac{\partial G}{\partial z}\right)_{z=1}=n K+\lambda \frac{1-K}{\alpha-\mu},
$$


and on substituting this into (2.20), we find that

$$
\rho(\tau)=\mathrm{e}^{-\tau / t_{c}}
$$

where

$$
t_{c}=\frac{1}{\alpha-\mu}>0
$$

is the autocorrelation time.

\subsection{Computation of the velocity of a single particle}

In this subsection, we will compute the mean particle velocity $\bar{u}_{p}$ as a function of the fluid velocity $u_{f}$. To that end, we will treat the rolling and saltating regimes separately. Any computation of the fluid forces acting on a particle poses the difficult question of how to properly define the fluid velocity field in the immediate vicinity of the particle (e.g. see Appendix A in Schmeeckle, Nelson \& Shreve 2007). Since in our subsequent applications (see $\S 4$ ), the flows are shallow, leading to fairly blunt velocity profiles in the cross-stream direction, we will not make any significant error in assuming that the reference fluid velocity is the mean velocity $\bar{u}_{f}=q_{w} / h$.

We now use the energy balance equation to compute the velocity of a single rolling particle, which is assumed to reach a steady regime nearly instantaneously once set in motion (Ancey et al. 2003). On average, for steady flow conditions, the power supplied by gravity and fluid drag force is entirely dissipated by contact forces

$$
m^{\prime} g \bar{u}_{p} \sin \theta+\bar{P}_{d}=\bar{P}_{c}
$$

where $m^{\prime}=m-4 \pi \rho_{f} a^{3} / 3$ is the buoyant mass, $\bar{P}_{d}=\overline{\boldsymbol{F}}_{d} \cdot \overline{\boldsymbol{u}}_{p}$ is the power of drag forces supplied to the particle, where $\overline{\boldsymbol{F}}_{d}=C_{d} \pi a^{2}\left|\boldsymbol{u}_{f}-\boldsymbol{u}_{p}\right|\left(\boldsymbol{u}_{f}-\boldsymbol{u}_{p}\right)$ is the drag force, with $C_{d}$ the drag coefficient. The power $P_{c}$ lost in contacts can be determined using results for a single bead rolling on a bumpy line in air. In this case, Ancey, Evesque \& Coussot (1996) have shown that dissipation can be broken into frictional and collisional parts

$$
\bar{P}_{c}=\kappa_{f} m^{\prime} g \bar{u}_{p} \cos \theta+\kappa_{c} \frac{\chi}{2 a} m \bar{u}_{p}^{3},
$$

where $(2 a)^{-1} \chi \bar{u}_{p}$ is the collision rate (if the bottom beads are regularly and closely arranged, then $\chi=1$ ), $\kappa_{c}=0.752$ reflects collisional dissipation, and $\kappa_{f}=0.104$ is a bulk friction coefficient. The resulting equation of motion is a second-degree polynomial in $\bar{u}_{p}$,

$$
m^{\prime} g \sin \theta+\frac{1}{2} \epsilon C_{d} \pi a^{2} \rho_{f}\left(\bar{u}_{p}-\bar{u}_{f}\right)^{2}=\kappa_{f} m^{\prime} g \cos \theta+\kappa_{c} \frac{\chi}{2 a} m \bar{u}_{p}^{2},
$$

where $\epsilon=+1$ when $\bar{u}_{p}<\bar{u}_{f}$ and $\epsilon=-1$ when $\bar{u}_{p}>\bar{u}_{f}$. The single physical solution is

$$
\bar{u}_{p}=k\left(S h^{\prime}\right) \bar{u}_{f},
$$

where

$$
\begin{aligned}
k\left(S h^{\prime}\right) & =\frac{1-\sqrt{D_{i}}}{1-4 \kappa_{c} r \chi /\left(3 \epsilon C_{d}\right)}, \\
D_{i} & =\frac{4}{9 S h^{\prime}} \frac{4 \kappa_{c} r \chi \cos \theta\left(\tan \theta-\kappa_{f}\right)+3 \epsilon C_{d}\left(\kappa_{c} r \chi S h^{\prime}-\cos \theta\left(\tan \theta-\kappa_{f}\right)\right)}{C_{d}^{2}},
\end{aligned}
$$


and where we have introduced the density ratio $r=\rho_{p} / \rho_{f}$. The parameter $k\left(S h^{\prime}\right)$ is expressed as a function of the modified Shields number

$$
S h^{\prime}=\frac{\bar{u}_{f}^{2}}{2(r-1) a g} .
$$

Analytical computation of the velocity of a saltating particle is more delicate, though examples have been given by a number of authors, including Gordon, Carmichael \& Isackson (1972), Abbott \& Francis (1977), van Rijn (1985), Wiberg \& Smith (1985), Niño \& Garcia (1994) and Ancey et al. (2002). To our knowledge, no analytic model provides realistic predictions of the trajectories and velocities of a saltating particle in water, because of the strong nonlinearities in the fluid forces exerted on the particle, and numerical models should be used to derive the velocity. By contrast, the experimental results are surprisingly simple: it is mostly possible to derive linear or power-law relations between the velocities and the flow conditions such as Shields number and shear velocity (van Rijn 1985). For the present purpose, it is best to use empirically derived analytical expressions rather than numerical models. Earlier experimental investigation in our two-dimensional flume showed that the mean velocity of a single particle can be expressed as

$$
\bar{u}_{p}=3.2\left(\bar{u}_{f}-u_{c r}\right),
$$

with $u_{c r}=1.25 \tan ^{1 / 2} \theta$ a critical velocity (Ancey et al. 2002).

It is possible to derive a single relation that holds for both rolling and saltating regimes (Ancey et al. 2003), but we do not develop this here.

\subsection{Summary}

Before addressing the validity of our approach by comparison with experiments, it is worth outlining its salient characteristics. For flows at equilibrium, we can compute the solid discharge as $\dot{n}=\sum_{i=1}^{N} u_{i} / L$. In the previous subsections, we have computed the number $N$ of particles in motion and their individual velocity $u_{i}$, which is assumed to be directly linked to the fluid velocity through (2.24) and (2.26). Under our model we have shown that $N$ is a random variable with the following properties.

(a) Its probability density is the negative binomial function (2.15) when particles are entrained both individually and collectively $(\mu>0)$, and has the Poisson form (2.17) when collective entrainment does not occur $(\mu=0)$.

(b) When $\mu>0$, the variance must exceed the mean, while they are equal when $\mu=0$, enabling us to determine whether collective entrainment takes place.

(c) The autocorrelation function of $N$ is the exponential function (2.21). As the particle velocity is not random, the solid discharge $\dot{n}$ must have the same autocorrelation as $N$;

(d) A particle continues moving for an exponential time, with parameter $\sigma$. The characteristic time (the mean duration of motion for one particle) is $t_{\sigma \mid 1}=1 / \sigma$.

Since particles move independently, the mean deposition rate when there are $n$ moving particles is $n \sigma$ and the associated characteristic time is denoted by $t_{\sigma}=1 /(n \sigma)$. The mean deposition rate is thus $\mathbb{E}(N) \sigma$. The lag times (i.e. the waiting between two successive deposition events within the window) must be distributed exponentially

$$
P\left(\Delta t_{m \rightarrow b}\right)=\frac{1}{t_{\sigma}} \exp \left(-\Delta t_{m \rightarrow b} / t_{\sigma}\right) .
$$

The number of deposition events within a time interval $\delta t$ can be computed similarly. The probability that a particle that moves at time $t$ settles by time $t+\delta t$ is 
$p_{d}=1-\exp [-(\sigma+v) \delta t]$. If $n$ particles are in motion at time $t$, the probability that $k$ particles settle within the time interval $[t, t+\delta t)$ is binomial with parameters $n$ and $p_{d}$; note that this holds true provided that $\delta t$ is chosen long enough for several depositions to occur, but sufficiently short for the number of particles that start moving and then settle within the same interval to be zero. It follows that the unconditional probability of observing $k$ particles that settle by $\delta t$ is negative binomial with parameters $r$ and $p^{\prime}=p /\left(p+p_{d}-p p_{d}\right)$ :

$$
\begin{aligned}
\operatorname{Prob}(k \text { depositions } ; \delta t) & =\sum_{n=k}^{\infty} \operatorname{Prob}(k \text { depositions } \mid n ; \delta t) \operatorname{Prob}(n) \\
& =\sum_{n=k}^{\infty} \operatorname{Bin}\left(k ; n, p_{d}\right) \operatorname{NegBin}(n ; r, p) \\
& =\operatorname{NegBin}\left(k ; r, p^{\prime}\right),
\end{aligned}
$$

since $\operatorname{Prob}(n)$ is the negative binomial distribution (2.15) with parameters $r=\lambda / \mu$ and $p=1-\mu / \alpha$ when $\mu>0$. When $\mu=0$, the resulting distribution is Poisson with parameter $r^{\prime \prime}=p_{d} r$. These expressions for the probability of observing $k$ settling particles could have been used to derive the probability of waiting times-for instance by using the classical arguments to show the duality between Poisson and exponential distributions (Cox \& Miller 1965)-and retrieve (2.27).

(e) The average fraction of particles that cross the control volume without coming to rest is $v /(\sigma+v)$, while a fraction $\sigma /(\sigma+v)$ of the particles stop within it.

(f) The entrainment coefficient $\lambda_{1}$ is expected to vary nonlinearly with the fluid velocity $\bar{u}_{f}$, but for narrow ranges of $\bar{u}_{f}$, the variation is almost linear.

$(g)$ When flows are at equilibrium, the model parameters $\lambda_{1}, \lambda_{0}, \mu, v$ and $\sigma$ must satisfy conditions (2.18) and (2.19). The autocorrelation time $t_{c}$ is given by (2.22). The ratio

$$
\frac{t_{c}}{t_{\sigma \mid 1}}=\frac{\sigma}{\sigma+v-\mu}
$$

is lower than unity when there is no collective entrainment $(\mu=0)$, but may exceed unity when there is collective entrainment $(\mu>0)$. Long-range correlation is the consequence of collective entrainment (memory effect due to the coupling between entrainment and the number of moving particles).

(h) The coefficients $\lambda_{1}, \mu$ and $\sigma$ represent the entrainment and deposition rates within the control volume $\mathscr{V}$ of length $L$. They are thus proportional to $L$.

\section{Experimental set-up and procedures}

\subsection{Overview}

We conducted our experiments in a narrow flume in which sediment consisted of glass beads of equal size. This is a simple laboratory representation of bed load transport, with the advantages that boundary conditions are perfectly controlled and a wealth of information can be obtained using imaging techniques. Figure 4 sketches the experimental facility. The nearly two-dimensional feature of this facility has some disadvantages: for instance, the low width-to-depth ratio leads to hydraulic difficulties since turbulence may be controlled by the sidewalls rather than the bottom; and using spherical particles of the same size can be problematic in terms of bed arrangement, thus producing artificial conditions for particle entrainment. This is, however, the price paid for convenient access to the detailed flows. 


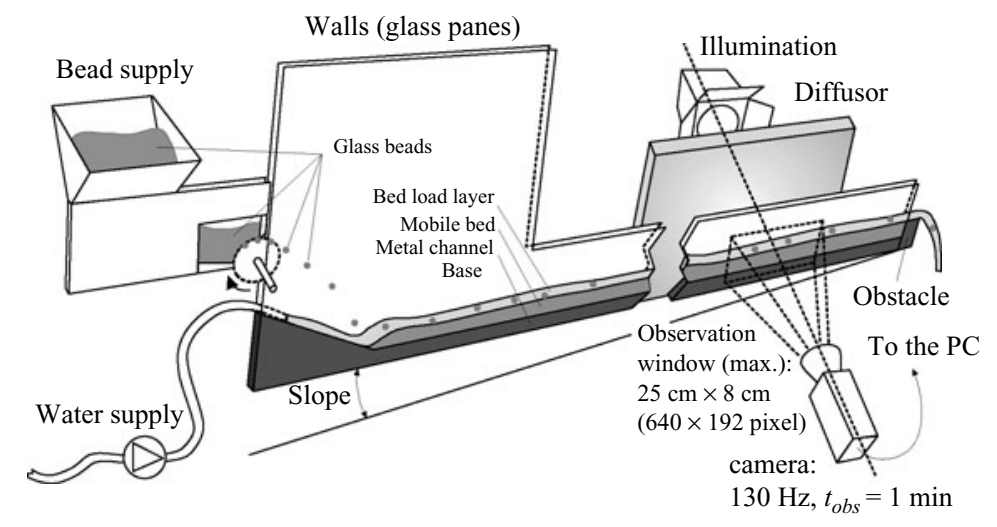

FIGURE 4. The experimental set-up.

We ran 15 experiments with different inclinations and various flow rates. Bed load equilibrium flows were achieved, as neither erosion nor deposition of particles occurred, averaged over sufficiently long time intervals. A short movie showing the motion of a few particles in our flume is available with the online version of the paper. Slopes and water discharges were selected to allow enough events for statistical analysis. The features of each run are summarized in tables 1 and 2. The hydraulic conditions are specified using classic dimensionless numbers. The flow Reynolds number is defined as $R e=4 R_{h} \bar{u}_{f} / v_{w}$, where $R_{h}=W h /(2 h+W)$ denotes the hydraulic radius, $\bar{u}_{f}=q_{w} / h$ the fluid velocity (averaged in the $y$-and $z$-directions), $v_{w}$ the kinematic viscosity of water, and $h$ the time-averaged water depth. The Froude number $F r=\bar{u}_{f} / \sqrt{g h}$ varied significantly over the experimental duration and along the main stream direction; its mean values are reported in tables 1 and 2 . The mean Froude number exceeded unity, which means that the flows were supercritical on average, but its instantaneous values fluctuated a great deal and frequent transitions to subcritical regimes occurred. This behaviour seems very close to that observed in gravel-bed rivers, where the Froude number also varies around the critical value (Grant 1997). We distinguished the solid discharge $\dot{n}_{0}$ imposed at the channel entrance (see $\S 3.2$ ) and the discharge $\dot{n}$ measured within the observation window. Note that we defined the solid discharge as the flux of beads per unit time. We can also define the solid discharge $q_{s}$ as the volume flow rate and relate it $\dot{n}_{0}$ using $q_{s}=\pi(2 a)^{3} \dot{n}_{0} / 6$. For $\dot{n}$, we used the same definition (2.3) as that used in the theoretical analysis. Tables 1 and 2 reported the values of $\dot{n}$ averaged over time (approximately $1 \mathrm{~min}$ ).

The solid concentration is defined as the ratio of the solid and the water discharges $C_{s}=q_{s} / q_{w}$. Values reported in tables 1-2 are low, indicating that particle flow was dilute. The ratio $h / d$ is low, typically in the range $0.8-7$. We used two definitions for the Shields number, which is classically defined as the ratio of the bottom shear stress to the stress equivalent of a buoyant force (Julien 1994),

$$
S h=\frac{\tau_{p}}{\left(\rho_{p}-\rho_{f}\right) g a},
$$

where $\tau_{p}$ is the bottom shear stress (here estimated as $\rho g h \sin \theta$ ). We also use the variant given by (2.25), which makes more sense for shallow flows. We report the mean particle velocities for the rolling $\left(\bar{u}_{r}\right)$ and saltating regime $\left(\bar{u}_{s}\right)$. We use $\kappa_{r / s}=N_{r} /\left(N_{r}+N_{s}\right)$ to refer to the number $N_{r}$ of particles in a rolling regime relative to the total number of moving particles $N=N_{r}+N_{s}$. 


\begin{tabular}{lcccccc}
\hline $\begin{array}{c}\text { Experiment } \\
\text { number }\end{array}$ & $\begin{array}{c}\text { E10-6 } \\
(\mathrm{e})\end{array}$ & $\begin{array}{c}\text { E10-7 } \\
(\mathrm{f})\end{array}$ & $\begin{array}{c}\text { E10-8 } \\
(\mathrm{g})\end{array}$ & $\begin{array}{c}\text { E10-9 } \\
(\mathrm{h})\end{array}$ & $\begin{array}{c}\text { E10-16 } \\
(\mathrm{i})\end{array}$ & $\begin{array}{c}\text { E10-21 } \\
(\mathrm{j})\end{array}$ \\
$\tan \theta(\%)$ & 10.0 & 10.0 & 10.0 & 10.0 & 10.0 & 10.0 \\
$\dot{n}_{0}\left(\mathrm{beads} \mathrm{s}^{-1}\right)$ & 5.3 & 6.7 & 8.0 & 10.0 & 15.4 & 20.0 \\
$q_{w}\left(10^{-3} \mathrm{~m}^{2} \mathrm{~s}^{-1}\right)$ & 4.15 & 4.42 & 5.38 & 5.54 & 8.19 & 10.31 \\
$h\left(\mathrm{~mm}^{2}\right)$ & 10.2 & 10.8 & 12.2 & 12.5 & 16.9 & 19.4 \\
$u_{f}\left(\mathrm{~m} \mathrm{~s}^{-1}\right)$ & 0.41 & 0.41 & 0.44 & 0.44 & 0.48 & 0.53 \\
$\dot{n}\left(\mathrm{beads} \mathrm{s}^{-1}\right)$ & 5.72 & 6.85 & 7.74 & 9.41 & 15.56 & 20.57 \\
$R e$ & 4020 & 4090 & 4550 & 4570 & 5280 & 5910 \\
$F r$ & 1.42 & 1.37 & 1.38 & 1.36 & 1.24 & 1.26 \\
$S h^{\prime}$ & 0.95 & 0.95 & 1.10 & 1.10 & 1.30 & 1.59 \\
$S h$ & 0.113 & 0.120 & 0.135 & 0.139 & 0.188 & 0.216 \\
$C_{s}(\%)$ & 2.40 & 2.69 & 2.50 & 2.96 & 3.30 & 3.47 \\
$h / d$ & 1.69 & 1.80 & 2.03 & 2.08 & 2.82 & 3.23 \\
$\bar{u}_{r}\left(\mathrm{~m} \mathrm{~s}^{-1}\right)$ & 0.063 & 0.074 & 0.065 & 0.075 & 0.075 & 0.072 \\
$\bar{u}_{s}\left(\mathrm{~m} \mathrm{~s}^{-1}\right)$ & 0.28 & 0.29 & 0.29 & 0.29 & 0.32 & 0.32 \\
$\kappa_{r / s}(\%)$ & 43.9 & 38.9 & 41.6 & 43.2 & 41.2 & 43.7 \\
\hline
\end{tabular}

TABLE 1. Flow characteristics and time-averaged values of dimensionless numbers characterizing bed load and water flow for $\tan \theta=10 \%$. Varying parameter: solid discharge $\dot{n}$. The notation E10-6 means: $\tan \theta=10 \%$ and $\dot{n} \approx 6$ beads $\mathrm{s}^{-1}$.

\begin{tabular}{|c|c|c|c|c|c|c|c|c|c|}
\hline $\begin{array}{l}\text { Experiment } \\
\text { number }\end{array}$ & $\begin{array}{l}\text { E7-6 } \\
\text { (a) }\end{array}$ & $\begin{array}{l}\text { E7-8 } \\
\text { (b) }\end{array}$ & $\begin{array}{c}\text { E7-9 } \\
\text { (c) }\end{array}$ & $\begin{array}{l}\text { E7-11 } \\
\text { (d) }\end{array}$ & $\begin{array}{c}\text { E12-9 } \\
(\mathrm{k})\end{array}$ & $\begin{array}{c}\text { E12-16 } \\
\text { (1) }\end{array}$ & $\begin{array}{c}\text { E12-21 } \\
(\mathrm{m})\end{array}$ & $\begin{array}{c}\text { E15-16 } \\
\text { (n) }\end{array}$ & $\begin{array}{c}\text { E15-21 } \\
\text { (o) }\end{array}$ \\
\hline $\tan \theta(\%)$ & 7.5 & 7.5 & 7.5 & 7.5 & 12.5 & 12.5 & 12.5 & 15.0 & 15.0 \\
\hline$\dot{n}_{0}\left(\right.$ beads $\left.\mathrm{s}^{-1}\right)$ & 5.7 & 7.8 & 8.7 & 10.9 & 9.3 & 15.2 & 20.0 & 15.6 & 21.5 \\
\hline$q_{w}\left(10^{-3} \mathrm{~m}^{2} \mathrm{~s}^{-1}\right)$ & 10.00 & 11.54 & 13.85 & 26.15 & 2.97 & 3.85 & 4.46 & 2.31 & 2.92 \\
\hline$h(\mathrm{~mm})$ & 18.9 & 20.8 & 24.9 & 40.8 & 7.0 & 8.2 & 9.4 & 4.9 & 6.7 \\
\hline$\sigma_{h}(\mathrm{~mm})$ & 2.2 & 2.3 & 2.5 & 2.8 & 2.2 & 2.3 & 2.4 & 2.0 & 2.5 \\
\hline $\bar{u}_{f}\left(\mathrm{~m} \mathrm{~s}^{-1}\right)$ & 0.53 & 0.55 & 0.56 & 0.64 & 0.42 & 0.47 & 0.48 & 0.47 & 0.44 \\
\hline$\dot{n}\left(\right.$ beads s $\left.^{-1}\right)$ & 5.45 & 7.76 & 9.20 & 10.99 & 9.52 & 15.52 & 19.86 & 15.45 & 20.55 \\
\hline$R e$ & 5860 & 6230 & 6400 & 7720 & 3760 & 4360 & 4600 & 3680 & 3830 \\
\hline$F r$ & 1.26 & 1.26 & 1.15 & 1.02 & 2.20 & 2.09 & 1.90 & 3.72 & 2.63 \\
\hline$S h$ & 0.158 & 0.173 & 0.207 & 0.340 & 0.098 & 0.114 & 0.130 & 0.082 & 0.111 \\
\hline$S h^{\prime}$ & 1.59 & 1.71 & 1.78 & 2.32 & 1.00 & 1.25 & 1.30 & 1.25 & 1.10 \\
\hline$C_{s}(\%)$ & 0.95 & 1.17 & 1.16 & 0.73 & 5.58 & 7.02 & 7.74 & 11.65 & 12.23 \\
\hline $\bar{u}_{r}\left(\mathrm{~m} \mathrm{~s}^{-1}\right)$ & 0.078 & 0.084 & 0.079 & 0.078 & 0.074 & 0.075 & 0.077 & 0.072 & 0.079 \\
\hline $\bar{u}_{s}\left(\mathrm{~m} \mathrm{~s}^{-1}\right)$ & 0.35 & 0.36 & 0.33 & 0.31 & 0.24 & 0.28 & 0.30 & 0.18 & 0.23 \\
\hline$\kappa_{r / s}(\%)$ & 31.2 & 30.3 & 33.3 & 32.4 & 78.4 & 72.6 & 63.9 & 95.1 & 82.2 \\
\hline
\end{tabular}

TABLE 2. Flow characteristics and time-averaged values of dimensionless numbers characterizing bed load and water flow. The notation E7-6 indicates: $\tan \theta \approx 7 \%$ and $\dot{n} \approx 6$ beads s$^{-1}$. See table 1 for the experiments at $\tan \theta=10 \%$.

\subsection{Channel, material used, and measurement system}

Experiments were performed in a tilted narrow glass-sided channel, $2 \mathrm{~m}$ in length and $20 \mathrm{~cm}$ in height. The channel width $W$ was adjusted to $6.5 \mathrm{~mm}$, which was slightly larger than the particle diameter $2 a$. In this way, particle motion was approximately two-dimensional and stayed in the focal plane of the camera. The channel slope $\tan \theta$ 
ranged from $5 \%$ to $15 \%$, but only experiments within the slope range $7.5-15 \%$ are reported here.

The channel base consisted of half-cylinders of equal size (radius $r=3 \mathrm{~mm}$ ) and randomly arranged on different levels. Disorder was essential, as it prevented slipping of entire layers of particles on the upper bed surface, which would have induced artificial erosion conditions. The effects of bed disorder were addressed by Böhm et al. (2004).

Coloured spherical glass beads with a nominal diameter of $2 a=6 \mathrm{~mm}$ and a density $\rho_{p}=2500 \mathrm{~kg} \mathrm{~m}^{-3}$ (provided by Sigmund Lindner GmbH, Germany) were injected from a reservoir into the channel using a wheel driven by a direct current motor and equipped with 20 hollows on the circumference (figure 4). For the experiments presented here, the injection rate $\dot{n}_{0}$ was 5-21 beads per second, with an uncertainty of less than $5 \%$. This corresponded to a solid discharge per unit width $q_{s} / W$ of 9 to $38 \times 10^{-5} \mathrm{~m}^{2} \mathrm{~s}^{-1}$. The water supply at the channel entrance was controlled by an electromagnetic flow meter provided by Krohne (France). The discharge per unit width $q_{w}$ ranged from 4 to $10 \times 10^{-3} \mathrm{~m}^{2} \mathrm{~s}^{-1}$.

The hydraulic conditions (velocity profile, bed friction, etc.) have been described in earlier papers (Ancey et al. 2002; Böhm et al. 2004). Although the flume was narrow, its hydraulic characteristics were like those observed in wide channels with shallow flows (Frey \& Reboud 2001).

The particles and the water stream were filmed using a Pulnix partial scan video camera (progressive scan TM-6705AN), placed perpendicular to the glass panes at $115 \mathrm{~cm}$ from the channel, approximately $80 \mathrm{~cm}$ upstream from the channel outlet. The camera was inclined at the same angle as the channel, behind which lights were positioned. An area of $L=22.5 \mathrm{~cm}$ in length and $8 \mathrm{~cm}$ in height was filmed and later reduced to accelerate image processing.

The camera resolution was $640 \times 192$ pixels for a frame rate of $129.2 \mathrm{f}$.p.s. (exposure time: $0.2 \mathrm{~ms}, 256$ grey levels). Each sequence comprised 8000 images because of limited computer memory; this corresponded to a duration of approximately 1 min. Images were analysed using the WIMA software, provided by the Traitement du Signal et Instrumentation laboratory in Saint-Étienne (France). Positions of the bead mass centres were detected by means of an algorithm combining several image-processing operations; particle trajectories were calculated using a tracking algorithm (Böhm et al. 2006).

\section{Experimental results}

With our experimental facility, we can probe quantities such as the number of particles that come to a halt or are entrained for some time interval. Figure 5 shows typical time series for four variables of interest: the number of particles passing from a resting to a moving state $(b \rightarrow m)$ and conversely $(m \rightarrow b)$; the solid discharge $\dot{n}$; the number of moving particles $N$; and the fluid velocity $\bar{u}_{f}$. These results were obtained for a mean bed slope of 0.1 and a solid discharge at the flume inlet $\dot{n}_{0}=8$ beads s$^{-1}$ (experiment 10-8 in table 1); the plots are typical of those for other solid discharges $\dot{n}_{0}$ and slopes. The bed was free of bedforms and therefore solid discharge fluctuations cannot be related to the collapse or evolution of organized bed structures.

Figure 6 shows the variations in the solid discharge $\dot{n}$ as a function of the mean fluid velocity $\bar{u}_{f}$. The solid discharge varies nearly linearly with the mean fluid velocity. A similar dependence is found if we replace $\bar{u}_{f}$ with the fluid discharge or the Shields number. The range of $\bar{u}_{f}\left(0.41-0.64 \mathrm{~m} \mathrm{~s}^{-1}\right)$ was, however, not sufficiently wide to claim 
(a)

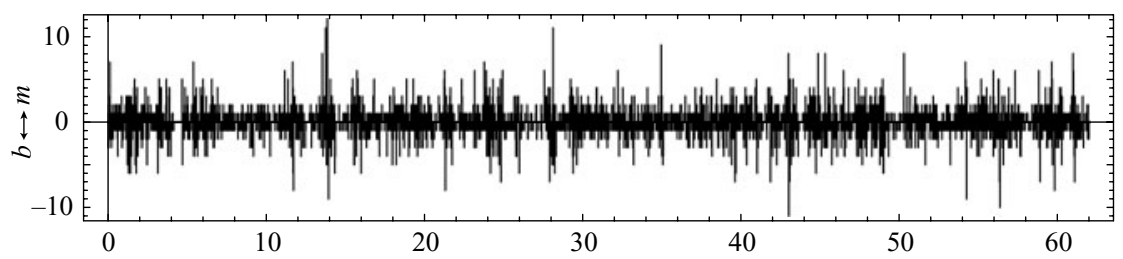

(b)

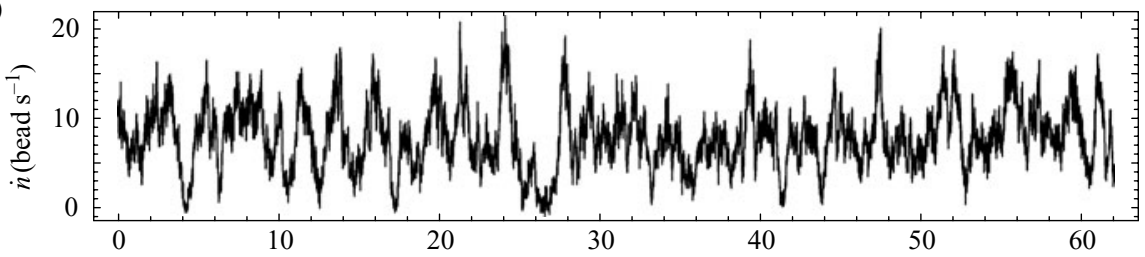

(c)

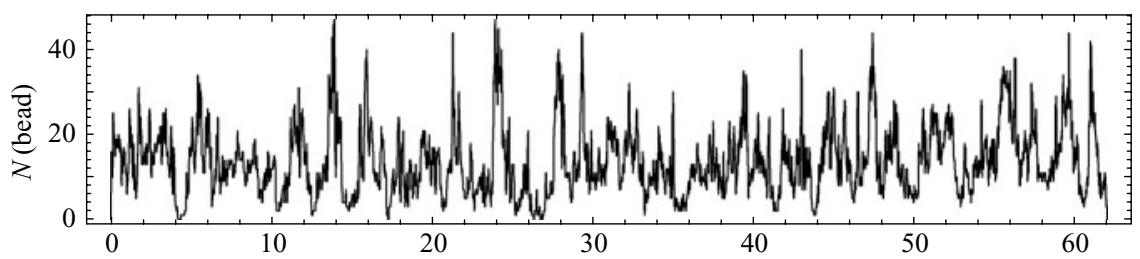

(d)

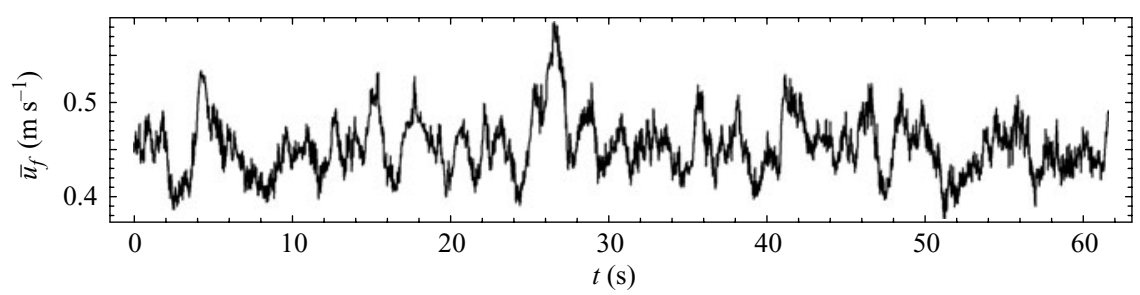

FigurE 5. Experiment (g) E10-8. (a) Particle exchanges between the bed and the flow: each bar oriented upward indicates the number of beads that passed from the resting state to the rolling regime over a given time interval $\delta t \approx 1 / 130 \mathrm{~s}$; downward-oriented bars represent the number of rolling particles coming to a halt. (b) Solid discharge $\dot{n}$ as a function of time. (c) Variation in the number of moving particles $N$. $(d)$ Mean fluid velocity $\bar{u}_{f}$ as a function of time.

that the relation $\dot{n}\left(\bar{u}_{f}\right)$ or $q_{w}$ was linear. Note the significant increase in the solid discharge when the bed slope is increased from $7.5 \%$ to $10 \%$, and the small changes when the slope is increased from $10 \%$ to $12.5 \%$. The strong dependence of solid discharge on channel inclination and the linearity with water flow rate are reflected by some empirical formulae. For instance, Rickenmann (2001) deduced from flume experiments representative of mountain streams that $q_{s} \propto \tan ^{2} \theta\left(q_{w}-q_{c}\right)$, with $q_{c}$ a critical water discharge corresponding to incipient motion. Incidentally, note that here, for the sake of simplicity, no correcting factor was applied to take into account sidewall effects, as is usually done in hydraulics for narrow channels; if we correct the influence of sidewalls on the mean fluid velocity by using the hydraulic radius rather than the flow depth, we obtain a smoother variation in $\dot{n}$ with bed slope (Frey et al. 2006).

Below we assess the reliability of our model by comparing its predictions with our experimental data. As the model is not closed, we must estimate some of its 


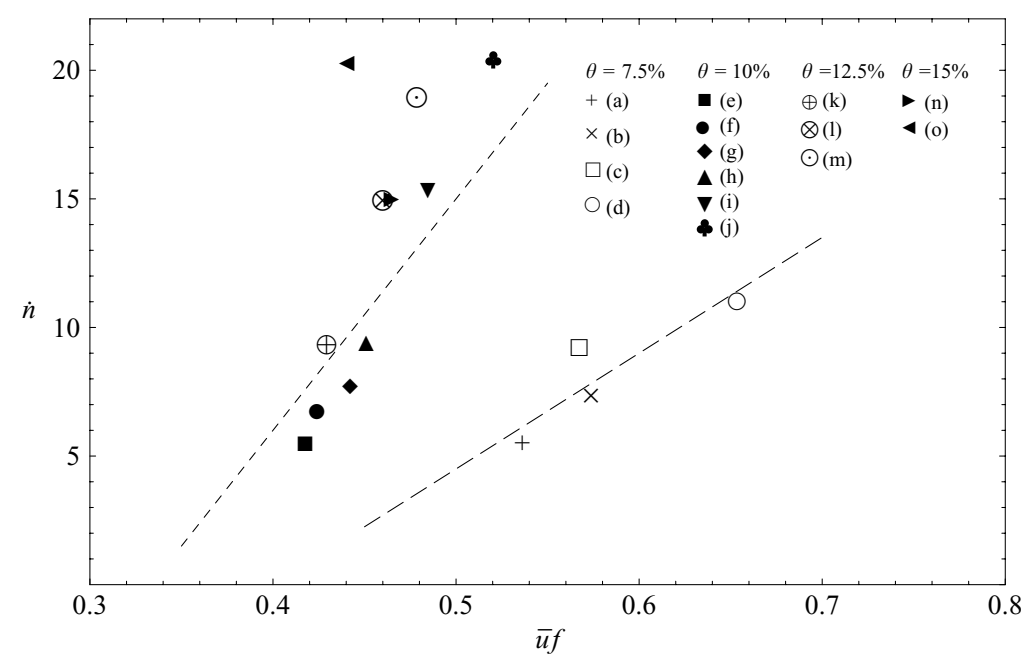

FIgURE 6. Variation in $\dot{n}$ with $\bar{u}_{f}$. The dashed lines represent empirical trends adjusted on the data: $\dot{n}=90 \bar{u}_{f}-30$ (dashed line) for $\tan \theta=0.1-0.125$ and $\dot{n}=45 \bar{u}_{f}-18$ (long dashed line) for $\tan \theta=0.075$.

parameters, which biases comparison to some degree. In $\S 4.1$, we outline the determination of the unknown parameters. In $\S 4.2$, we analyse the probability distribution and the autocorrelation function of the number of moving particles. Although there is good agreement between theory and experiments as regards the probability distribution function of $N$, there is an appreciable difference for the autocorrelation time $t_{c}$, perhaps as a consequence of collective effects when particles come to rest (§4.3). In $\S 4.4$, we investigate the dependence of the entrainment rates on the mean fluid velocity. A striking result emerges in the analysis of the mean particle velocity ( $(4.5)$ : we show that $\bar{u}_{p}$ exhibits little dependence on $\bar{u}_{f}$, which contrasts with physical intuition and theoretical analysis $(\S 2.5)$. Finally, in $\S 4.6$, we try to estimate the different sinks of energy for flows at equilibrium.

\subsection{Parameter estimation}

A classic impediment to any approach to sediment transport is that the models contain parameters that cannot be computed analytically, because they are empirical or represent complex processes whose description requires heavy computation. Our model involves eight variables: the number of moving particles $N$, the mean particle velocity $\bar{u}_{p}$, the solid discharge $\dot{n}$, the inflow rate $\lambda_{0}$, the entrainment rate of individual particles $\lambda_{1}$, the collective entrainment rate $\mu$, the deposition rate $\sigma$, and the outflow rate $\nu$. The fluid discharge $q_{w}$ and the channel slope $\theta$ are control parameters of our experiments. These variables are not independent (see §2.6), as we have found five relationships among the eight parameters related to the solid discharge; thus we would require three additional relations to close our system. Here, the closure equations are replaced with data fitting on three variables. Since we tuned the solid flow rate at the channel entrance for the flow to be at equilibrium, we consider that the flow rate and the number of particles are prescribed. Moreover, we can measure the settling rate to deduce $\sigma$. This provides us with the three required additional equations. We therefore assume below that $\lambda_{0}, N$ and $\sigma$ are prescribed at the channel inlet or measured within the control volume. From the record of $N(t)$, we infer the mean $\bar{N}$ and the variance $\operatorname{Var} N$. The deposition rate $\sigma$ was computed as the ratio of 


\begin{tabular}{cccccccccccc}
\hline Number & $\bar{N}$ & $\operatorname{Var} N$ & $\hat{t}_{c}$ & $\tilde{t}_{c}$ & $\lambda_{0}$ & $\lambda_{1}$ & $v$ & $\sigma$ & $\mu$ & $E$ & $\hat{E}$ \\
(a) & 7.06 & 22.53 & 0.59 & 0.36 & 5.45 & 6.59 & 0.77 & 4.67 & 3.74 & 33.3 & 33.0 \\
(b) & 9.83 & 30.94 & 0.52 & 0.27 & 7.76 & 11.21 & 0.79 & 5.28 & 4.14 & 51.7 & 51.9 \\
(c) & 12.19 & 35.43 & 0.50 & 0.29 & 9.20 & 15.14 & 0.75 & 5.05 & 3.81 & 61.8 & 61.5 \\
(d) & 14.86 & 38.51 & 0.46 & 0.23 & 10.99 & 21.40 & 0.74 & 4.91 & 3.47 & 72.4 & 72.9 \\
(e) & 9.46 & 70.74 & 1.30 & 0.37 & 5.72 & 1.53 & 0.60 & 5.13 & 4.96 & 47.9 & 48.5 \\
(f) & 9.86 & 36.89 & 0.67 & 0.36 & 6.85 & 7.80 & 0.69 & 4.86 & 4.07 & 48.5 & 47.9 \\
(g) & 13.76 & 64.55 & 0.85 & 0.24 & 7.74 & 8.42 & 0.56 & 4.95 & 4.34 & 67.9 & 68.1 \\
(h) & 13.67 & 49.09 & 0.66 & 0.33 & 9.41 & 11.24 & 0.69 & 4.74 & 3.91 & 65.0 & 64.7 \\
(i) & 22.83 & 72.09 & 0.61 & 0.21 & 15.56 & 22.07 & 0.68 & 4.52 & 3.56 & 102.7 & 103.2 \\
(j) & 34.21 & 128.91 & 0.75 & 0.17 & 20.57 & 24.78 & 0.60 & 4.39 & 3.67 & 151.2 & 150.3 \\
(k) & 21.89 & 123.38 & 1.07 & 0.26 & 9.52 & 10.96 & 0.43 & 4.84 & 4.34 & 106.4 & 105.9 \\
(l) & 32.39 & 182.68 & 1.07 & 0.20 & 15.52 & 14.64 & 0.48 & 4.77 & 4.32 & 154.7 & 154.6 \\
(m) & 38.52 & 222.88 & 1.15 & 0.23 & 19.86 & 13.62 & 0.52 & 4.51 & 4.16 & 173.0 & 173.9 \\
(n) & 43.31 & 214.31 & 1.08 & 0.17 & 15.45 & 24.49 & 0.36 & 4.21 & 3.64 & 182.4 & 182.2
\end{tabular}

TABLE 3. Measured values of the total number of particles moving within the window (mean values $\overline{\bar{N}}$ and variances $\operatorname{Var} N$ ), autocorrelation times $\hat{t}_{c}$ computed using (2.22), autocorrelation times $\tilde{t}_{c}$ estimated by fitting an exponential curve to the data, and inferred values of the model parameters obtained using (4.1). Times in $\mathrm{s}$, rates in beads $\mathrm{s}^{-1}$, entrainment coefficients in $\mathrm{s}^{-1}$. Comparison between the measured entrainment rate $E$ and estimated value $\hat{E}=\lambda_{1}+\mu \bar{N}$.

the total number of particles deposited to the experimental duration (approximately $1 \mathrm{~min})$.

We can then estimate the coefficients $\mu, v$ and $\lambda_{1}$ using (2.16) and the equilibrium conditions (2.18)-(2.19), yielding

$$
\mu=\lambda_{0}\left(\frac{1}{\bar{N}}-\frac{1}{\operatorname{Var} N}\right)+\sigma\left(1-\frac{\bar{N}}{\operatorname{Var} N}\right), \quad \lambda_{1}=\left(\lambda_{0}-\bar{N} \sigma\right) \frac{\bar{N}}{\operatorname{Var} N}-\lambda_{0}, \quad \bar{N} v=\lambda_{0} .
$$

The autocorrelation time $\hat{t}_{c}$ of $\dot{n}(t)$ and $N(t)$ is given by (2.22). In practice, the autocorrelation function was estimated by taking the Fourier transform of $N(t)$

$$
\tilde{\rho}=\mathscr{F}^{-1}\left(\mathscr{F}(N) \mathscr{F}(N)^{*}\right),
$$

where $\mathscr{F}$ denotes the Fourier transform, $\mathscr{F}(N)^{*}$ is the complex conjugate of $\mathscr{F}(N)$, and $\mathscr{F}^{-1}$ denotes the inverse Fourier transform. Another way to estimate the autocorrelation time is to fit the exponential form (2.21) directly to the data to estimate the experimental correlation time $\tilde{t}_{c}$. The estimate depended on the number of observations used to fit the exponential function. For instance, for experiment (g) E10-8, we obtained $\tilde{t}_{c}=0.283 \mathrm{~s}$ when taking the first 50 data points against $\tilde{t}_{c}=0.243 \mathrm{~s}$ when a row of 100 data points was used. The empirical autocorrelation function may become negative.

The parameter estimates are reported in table 3 . This table also shows the measured entrainment rate $E$ and its estimated value $\hat{E}=\bar{N} \mu+\lambda_{1}$, computed from the estimates of $\mu$ and $\lambda_{1}$. The perfect match confirms that the flows were at equilibrium; $\hat{E}$ was in fact obtained from the measurement of the settling rate coefficient $\sigma$ using (4.1).

\subsection{Number of moving particles}

Figure 7 shows the probability density function of $N$ for experiments (b), (e), (k) and (n); refer to the supplement available with the online version of the paper to see all the plots. The dots represent the empirical probabilities, while the dashed 

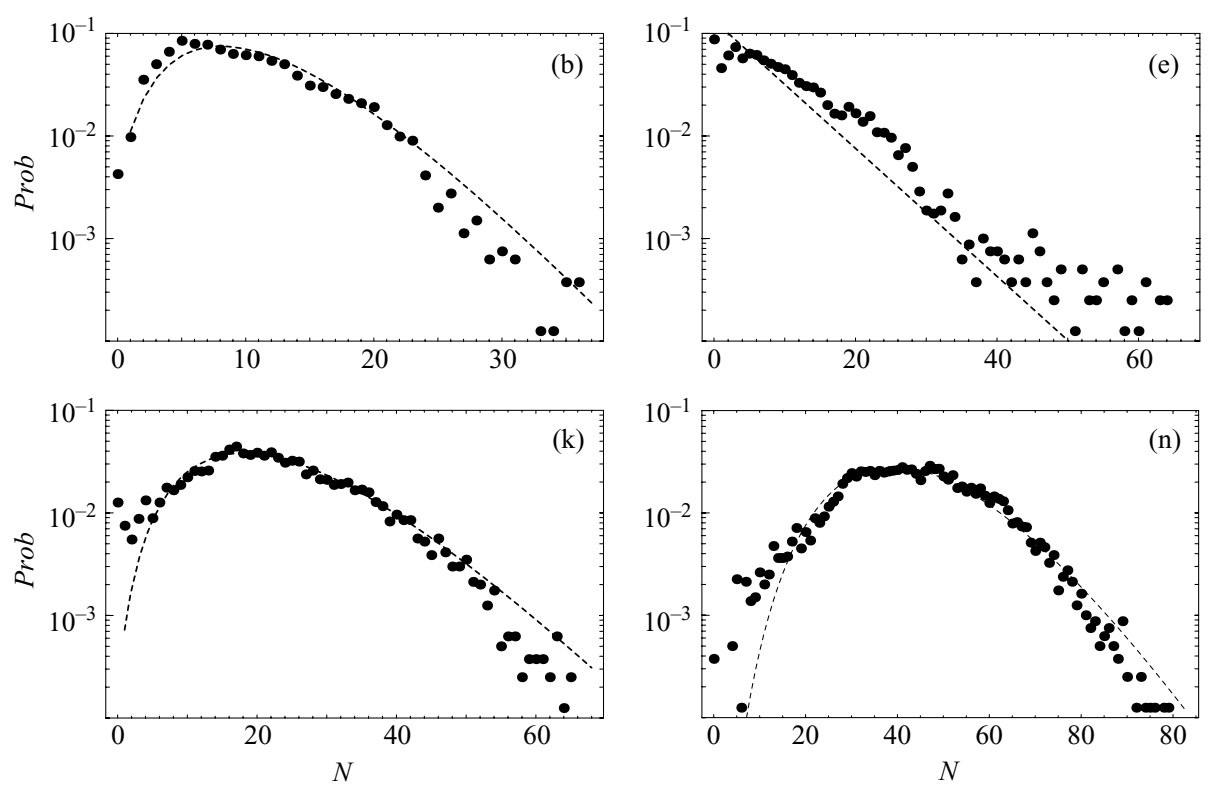

FIGURE 7. Empirical probability density of the total number of moving beads $N$ (black dots). The dashed line is the probability density function of the negative binomial distribution. Experiments (b), (e), (k) and (n).

line stands for the negative binomial distribution (2.15), the parameters of which were estimated using the measured values $\operatorname{Var} N$ and $\bar{N}$ reported in table 3; for the sake of readability, we plotted the discrete probability mass functions as continuous curves.

First note the fairly good agreement between the data and theoretical probability distribution for all experiments. Local departures and data scattering are seen, but they are usually associated with low probabilities. Since 8000 data points were used for each run, empirical probabilities lower than $10^{-3}$ are unimportant. Deviations are also visible at $N=0$, but it should be kept in mind that algorithmically, it was difficult to discriminate cases in which a particle was really set in motion from those in which a bed particle merely swung around its rest position (Böhm et al. 2006), and this might introduce slight errors when counting the number of moving particles. The plots confirm that the negative binomial distribution is a good candidate for describing the statistical behaviour of $N$ over a wide range of flow conditions. If we set $\mu=0$ (Einstein-like theory), the theoretical distribution is Poisson and has equal variance and mean. This supports the idea of collective entrainment or, at least, does not undermine the simple assumption used for modelling entrainment.

There is a significant change in the distribution shape when the water discharge increases. When it is low (e.g. (e) in figure 7), the empirical distribution is close to a straight line on a logarithmic scale, implying that the number of particles decays exponentially within the observation window. For experiment (e), the discrepancy between empirical and theoretical probability distributions is the most pronounced of all runs, which may mean that theory performs less well for these discharges (as expected, see §2.2). With increasing water discharge, the probability distribution takes a bell shape, which is first skewed, then nearly symmetric at the highest slopes and 

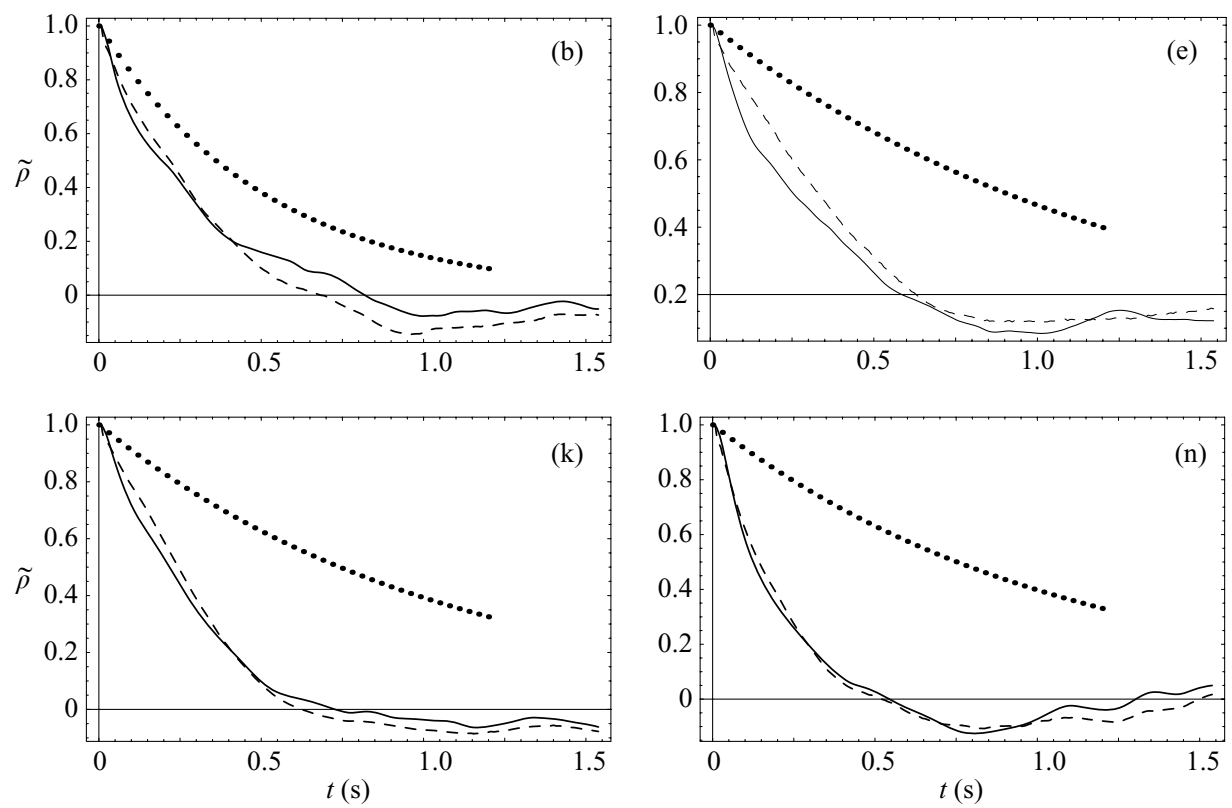

FIGURE 8. Autocorrelation functions of the number of moving beads (solid line) and the solid discharge (dashed lines). Dotted lines stand for the theoretical autocorrelation function (2.22), when the autocorrelation time $\hat{t}_{c}$ is evaluated using (4.1). Experiments (b), (e), (k) and (n).

flow rates (see (n) of figure 7). In the case of run (n), the discrete empirical probability distribution can be approximated by a Gaussian distribution.

For each run, the sample variance exceeded the mean, but the $\operatorname{ratio} \operatorname{Var} N / \bar{N}$ decreased with increasing water discharge (or Shields number). For instance, for slope $\tan \theta=0.1$, the ratio $\operatorname{Var} N / \bar{N}$ was as high as 7 for run (e) and dropped to below 3 for run (o). Thus for each run, the number of moving particles varied frequently and widely, but with increasing water discharges, wide fluctuations became less frequent, which substantiates the idea that at high water discharges, sediment transport becomes more continuous.

Figure 8 shows the autocorrelation functions of the total solid discharge, the number of moving beads, and the theoretical curve (2.22), where the autocorrelation time $t_{c}$ is replaced by its estimate $\hat{t}_{c}$ given by (4.1). The autocorrelation functions $\tilde{\rho}$ for the solid discharge and the number of moving particles are similar for each run, as theoretically expected (see $\S 2.6$ ), which shows that the instantaneous particle velocities were never too far from their mean values. The behaviour of $\tilde{\rho}$ at short times is nearly exponential, as expected, but the theoretical autocorrelation time $\hat{t}_{c}$ is much longer than the experimental value $\tilde{t}_{c}$. The values of $\hat{t}_{c}$ and $\tilde{t}_{c}$ given in table 3 show that $\hat{t}_{c} / \tilde{t}_{c}$ ranges from 1.6 to 6.3 , with a mean value of 3.5 . We shall see that to a large extent, this discrepancy stems from the statistical behaviour of deposition events (see $\S 4.3$ ), which are more frequent than theoretically computed, but of smaller amplitude over a time interval $\delta t$.

Despite this shortcoming of the theoretical model, the salient features of soliddischarge fluctuations are correctly described, notably the ratio $t_{c} / t_{\sigma}$ (see $\S 2.6$ ) ranges from 2 to 9, which shows that the solid discharge exhibited long-range fluctuations, the durations of which are much longer than the typical time scale associated with particle motion. 

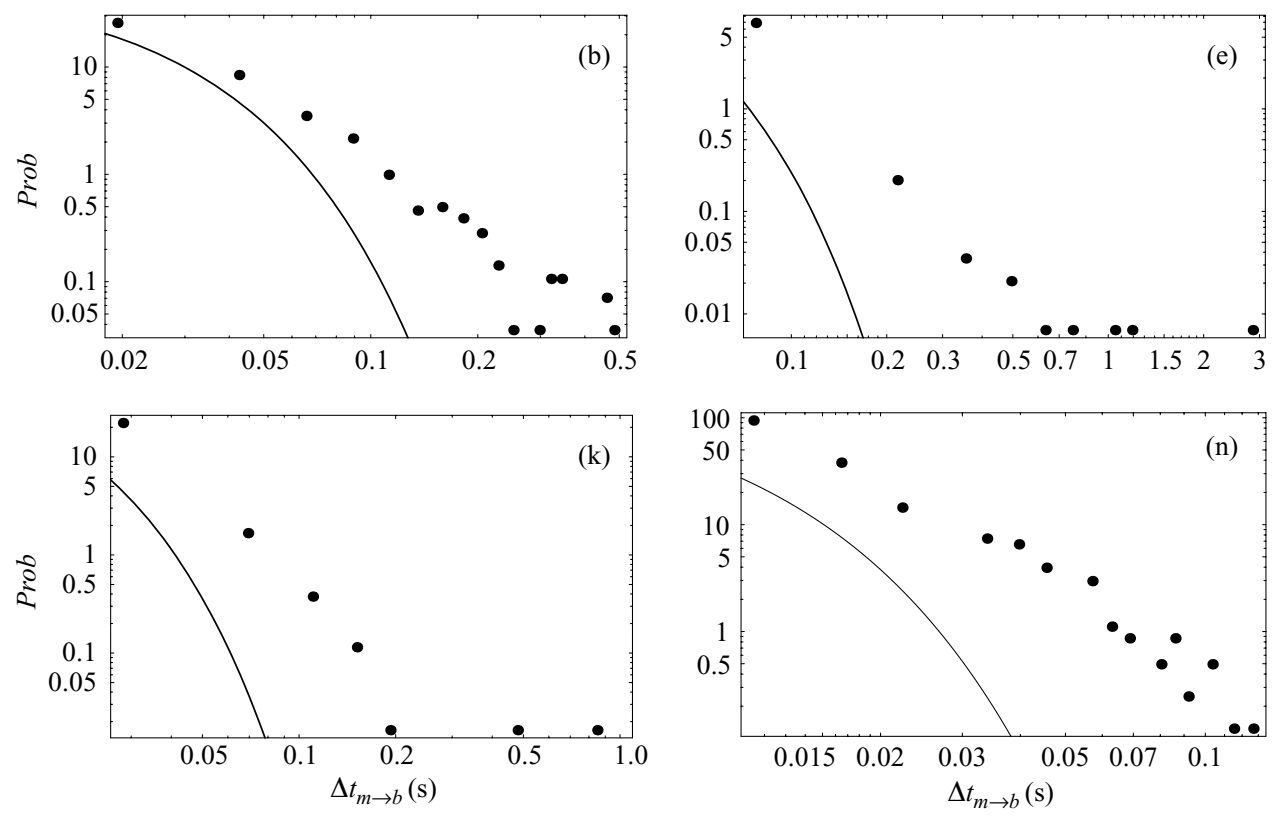

FIGURE 9. Probability distribution of lag times: dots represent empirical probabilities, while the solid line stands for the theoretical curve (2.27). Experiments (b), (e), (k) and (n).

\subsection{Deposition rate}

Another aspect of our experiments is the deposition rate of moving particles. From time series such as that in figure 5(a), we can compute the lag times $\Delta t_{b \rightarrow m}$ between two deposition events within the observation window, and hence infer the statistical properties of the lag times $\Delta t_{b \rightarrow m}$. Figure 9 shows the empirical probability distribution of $\Delta t_{b \rightarrow m}$ for runs (b), (e), (k), (n) (refer to the online supplement to see all the plots).

We also plot the theoretical curve given by (2.27), which is an exponential density with parameter $t_{\sigma}^{-1}=(1-p) r \sigma / p=\bar{N} \sigma$. As in $\S 4.2$, given the size of our samples, the empirical probabilities make sense only when they are in excess of $10^{-3}$; moreover, since the acquisition rate was approximately $130 \mathrm{~Hz}$, we could not resolve events that occurred within time intervals shorter than $10 \mathrm{~ms}$.

For low flow rates and gentle slopes $(\tan \theta \leqslant 0.1)$, the theoretical probability distribution provides a crude approximation to the empirical distribution: while the shape of the empirical distribution is well captured (exponential behaviour), the deviation between the theoretical and empirical curves suggests that the mean lag time is longer than predicted. At higher flow rates or steep slopes, the model yields even cruder approximations, but still captures the main trend. The deviation between theoretical and experimental curves is more pronounced for experiments conducted at $\tan \theta=0.15$. Figure 10 compares the expected mean lag time $t_{\sigma}$ with the mean of the experimental samples $\Delta \bar{t}_{m b}$. On the whole, the agreement is good, but a closer look shows that the mean experimental lag time is twice the expected value $t_{\sigma}$ : empirical deposition events occur more frequently than assumed with our model.

If we plot the probability of observing $n_{m \rightarrow b}$ particles settling during a time interval $\delta t=1 / 130 \mathrm{~s}$, there are smaller differences between the theoretical and empirical distributions. Figure 11 shows these probability distributions for runs (b), (e), (k) and 


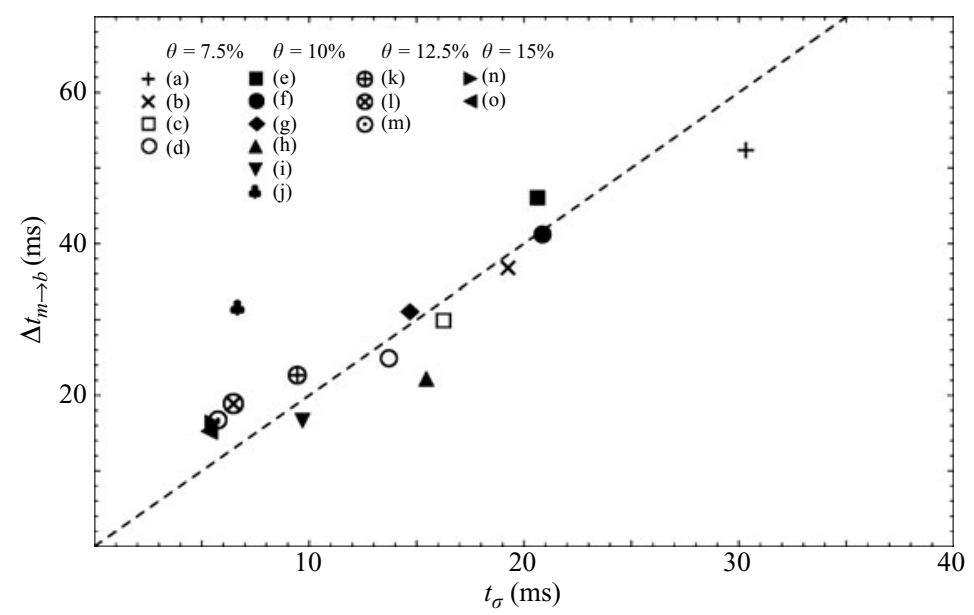

Figure 10. Comparison between the mean experimental lag time $\Delta \bar{t}_{m b}$ and the theoretical value $t_{\sigma}=1 /(\bar{N} \sigma)$. The dashed line stands for the empirical trend $\Delta \bar{t}_{m b}=2 t_{\sigma}$.
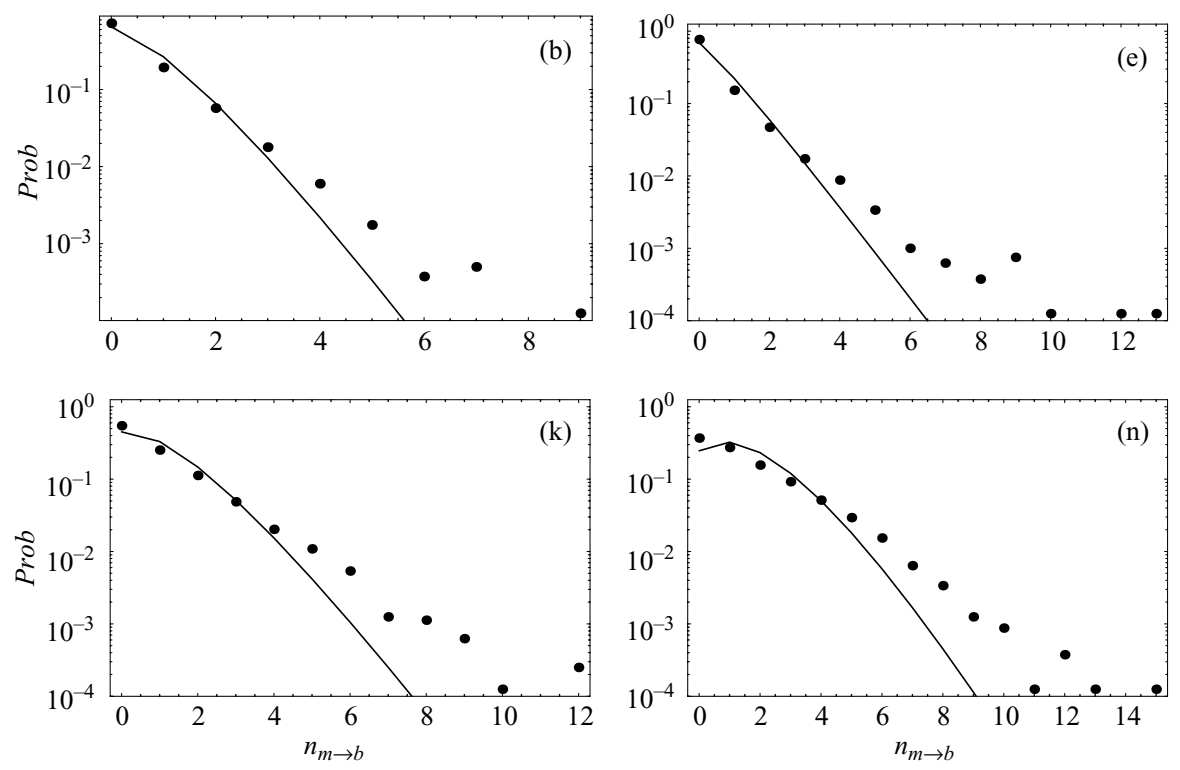

FiguRE 11. Probability distribution of the number of particles that come to a halt during a time interval $\delta t$ : dots represent empirical probabilities, while the dashed line represents the theoretical distribution (2.28). Experiments (b), (e), (k) and (n).

(n) (refer to the online supplement to see all the plots). Theoretically, the number of settling particles follows the probability distribution (2.28) derived in $\S 2.6$. The behaviour at low $n_{m \rightarrow b}$ values is captured well by the theoretical curve: for all slopes and flow rates, the data fall onto the theoretical curve, for $n_{m \rightarrow b}$ as large as 3 to 6 . For larger $n_{m \rightarrow b}$ values, there are significant deviations between data and predictions. Experimentally, we observe an exponential decay of the number of settling particles, which shows that occasionally, a group of several particles (up to 12-14 particles at the steepest slopes) can come to rest. The exponential decay is observed for all 
slopes and discharges. The theoretical probability also entails exponential decay, but its decay rate is much larger (by a factor of roughly 10) than the empirical rate.

In conclusion, we observe empirically that deposition occurs a little less frequently and the number of deposited particles over short time intervals can be much larger than expected with our model. This higher activity contributes to decreasing the autocorrelation time $t_{c}$ of the solid discharge, which may explain why we found only the order of magnitude of $t_{c}$. Note also that table 3 shows little correlation between the deposition rate coefficient $\sigma$ and the fluid velocity $\bar{u}_{f}$, which may suggest that $\sigma$ is a constant parameter.

\subsection{Entrainment rates}

Figure 12 shows how the entrainment coefficients $\lambda_{1}$ and $\mu$ vary with the mean fluid velocity $\bar{u}_{f}$ and how $\lambda_{0}$ is related to $\lambda_{1}$. Note that the corresponding mean entrainment rates are $\lambda_{1}$ and $\mu \bar{N}$ (and not $\mu$ alone).

Figure $12(a)$ reveals a linear relation between the inflow coefficient $\lambda_{0}$ and the entrainment rate $\lambda_{1}$ for the gentlest slopes and lowest flow rates $(\tan \theta \leqslant 0.1$ and $\dot{n}<16$ beads s$^{-1}$ ). At higher flow rates or for steep slopes, there is no clear trend, but this may stem from the dearth of data. At very low entrainment rates, the influx coefficient $\lambda_{0}$ is much higher than $\lambda_{1}$, suggesting that bed load transport can take place with little entrainment of isolated particles.

Figure $12(b)$ shows that the entrainment rate $\lambda_{1}$ grows with increasing fluid velocity. There is no very clear trend, but it appears that the data related to slopes $\tan \theta=0.1$ and $\tan \theta=0.125$ collapse onto a single straight line, while the data corresponding to $\tan \theta=0.075$ form a parallel line. This closely reflects the behaviour observed for the solid discharges in figure 6 , with the same reservation about the role of sidewalls. This strong dependence on slope suggests that theoretical analysis of incipient motion on sloping beds, which provides weak dependence on $\cos \theta$, underestimates the influence of bed slope on entrainment. The linear variation in $\lambda_{1}$ with $\bar{u}_{f}$ is consistent with the heuristic arguments presented in $\$ 2.2$. The estimated waiting time given by (2.11) is around $50 \mathrm{~ms}$ if we take the typical values $P_{c}=0.5, L_{x} \sim h=2 \mathrm{~cm}$, and $\langle u\rangle \sim \bar{u}_{f}=50 \mathrm{~cm} \mathrm{~s}^{-1}$, consistent with the mean lag time values reported in figure 10 (since $\Delta t_{m \rightarrow b} \approx \Delta t_{b \rightarrow m}$ in a steady state). For the highest bed slope $\tan \theta=0.15$, we observe a drop in the entrainment rate with increasing fluid velocity, which is probably directly linked to the very low flow submergence conditions for this slope.

Figure $12(c)$ shows that the collective entrainment coefficient $\mu$ decreases nearly linearly with increasing fluid velocity, but this decay rate is very low since it passes from 4 bead s s${ }^{-1}$ to 3 bead s s${ }^{-1}$ when the mean fluid velocity is increased from 0.4 to $0.65 \mathrm{~m} \mathrm{~s}^{-1}$. It may be reasonable to consider it as a constant parameter.

\subsection{Particle velocity}

Figure 13 shows how the mean particle velocity varies with fluid velocity for particles in a rolling regime (figure 13a) or in a saltating regime (figure 13b). It is striking that the mean velocity $\bar{u}_{r}$ of rolling particles is almost independent of fluid velocity in our experiments, while theoretically we obtained a nonlinear increase in $\bar{u}_{r}$ with $\bar{u}_{f}$ (see $\left.\S 2.5\right)$ in full agreement with earlier observations, when a single particle was set in motion on a fixed rough bed (Ancey et al. 2003). Moreover, the order of magnitude of $\bar{u}_{r}$ predicted by our theory is approximately $20 \mathrm{~cm} \mathrm{~s}^{-1}$ for $\tan \theta=0.1$, while experimentally, $\bar{u}_{r}$ was closer to $6 \mathrm{~cm} \mathrm{~s}^{-1}$.

The same observation applies to the saltating particles, whose mean velocity exhibits little correlation with the mean fluid velocity. Contrary to the rolling velocity, the semi-empirical relation (2.26) intersects the data, but the dependence of the saltating velocity $\bar{u}_{s}$ on $\bar{u}_{f}$ is much more pronounced theoretically than experimentally. 

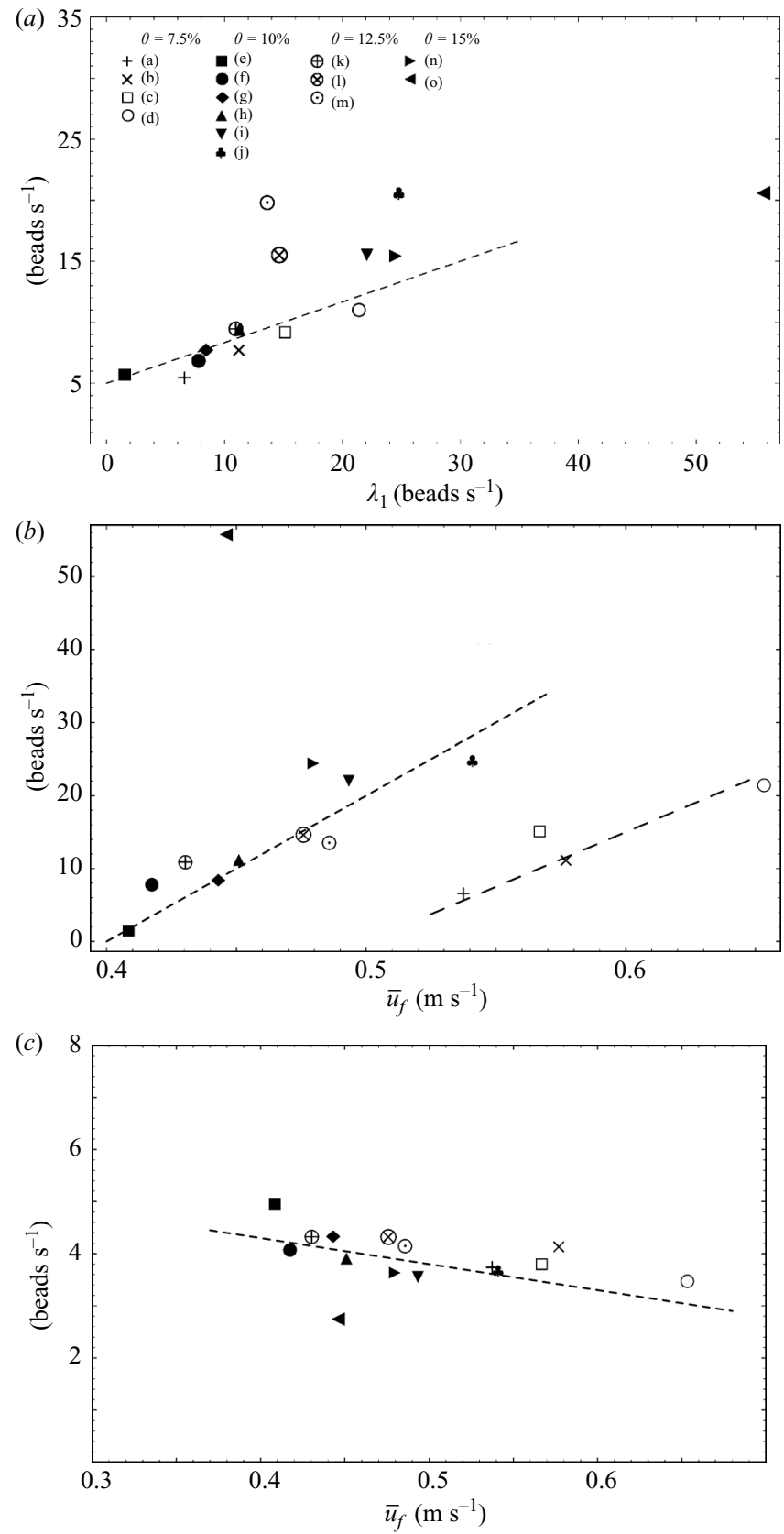

FiguRE 12. (a) $\lambda_{0}$ as a function of $\lambda_{1}$; the dashed line represents the experimental trend $\lambda_{0}=5+\lambda_{1} / 3+5$. (b) Entrainment rate $\lambda_{1}$ as a function of the mean fluid velocity; the dashed line represents the experimental trend $\lambda_{1}=200\left(\bar{u}_{f}-0.4\right)$ for slopes $\tan \theta \geqslant 0.1$, while the long-dashed line stands for the experimental trend $\lambda_{1}=150\left(\bar{u}_{f}-0.5\right)$. (c) Entrainment coefficient $\mu$ as a function of the mean fluid velocity; the dashed line represents the experimental trend $\mu=2.3-5 \bar{u}_{f}$.

Although the flows were dilute, there was no clear relation between the mean velocity of one particle of a system of $N$ moving particles and the mean velocity of an isolated particle, which was entrained alone by a turbulent water stream. For the rolling regime, this may be easily understood: because the rolling particles 
(a)

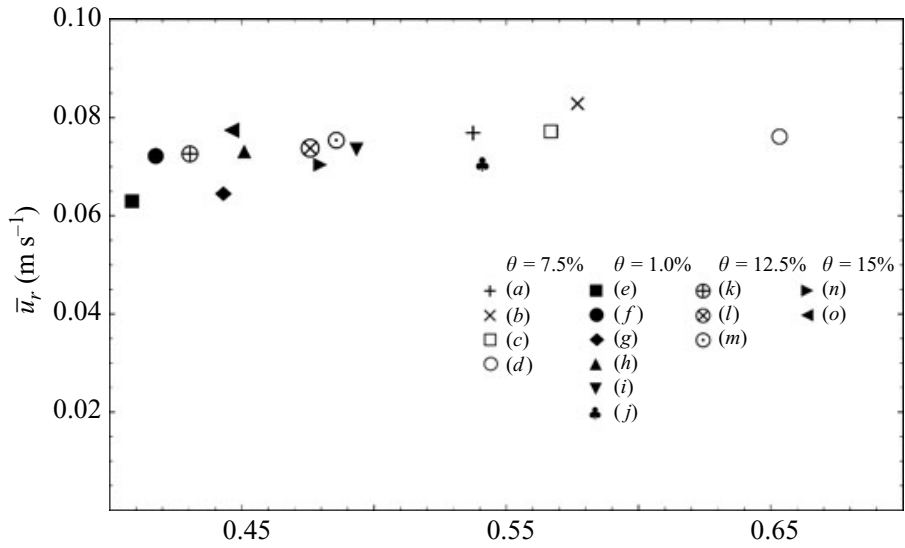

(b)

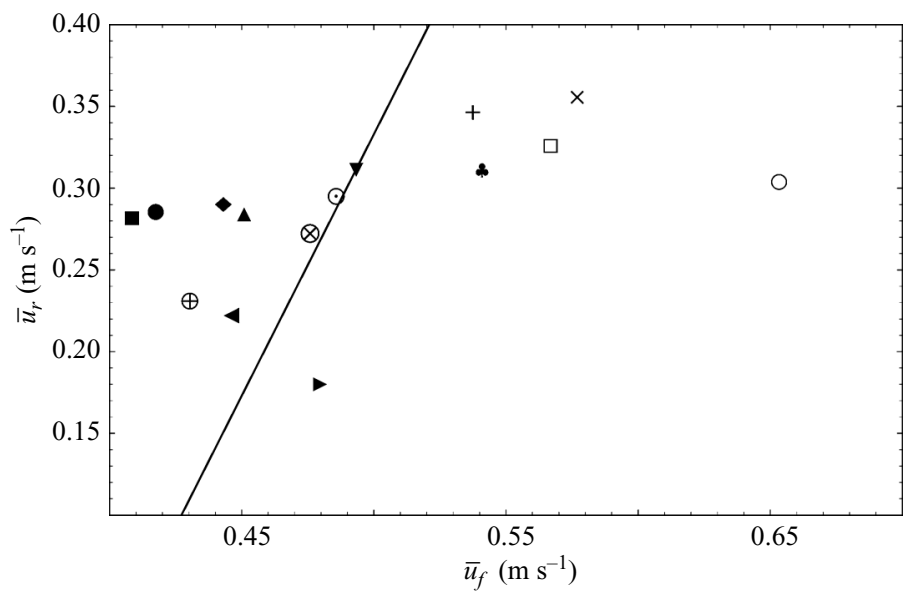

FIGURE 13. (a) Variation of the rolling velocity with the mean fluid velocity. (b) Variation of the saltating velocity with the mean fluid velocity; the solid line represents the velocity of a single isolated particle, as predicted by (2.26) for $\tan \theta=0.1$.

moved on a mobile bed, the path was less regular in our bed-load experiments than in our earlier experiments, where a single particle moved down a corrugated stationary bottom. This probably induced an increase in the energy dissipation. What is difficult to understand, however, is the very weak dependence of $\bar{u}_{s}$ on $\bar{u}_{f}$. A plausible explanation is that in our shallow turbulent flows, particle trajectories were substantially influenced by the wakes induced by neighbouring particles; this wake effect is probably less pronounced in thick flows.

This weak dependence has important consequences for the computation of the solid discharge $\dot{n}$. Recall that $\dot{n}=\sum_{i=1}^{N} u_{i} v_{p} / L$, with $v_{p}$ the particle volume; if the particle velocity $u_{i} \approx \bar{u}_{p}$ is nearly independent of $\bar{u}_{f}$, then we can approximate

$$
\dot{n} \approx \frac{N v_{p}}{L} \bar{u}_{p},
$$

and the dependence of $\dot{n}$ on $\bar{u}_{f}$ is entirely contained in $N$. This contradicts the common assumption in Bagnold-like models, where the mismatch between the mean fluid and particle velocities is the control parameter of momentum transfers and thus 


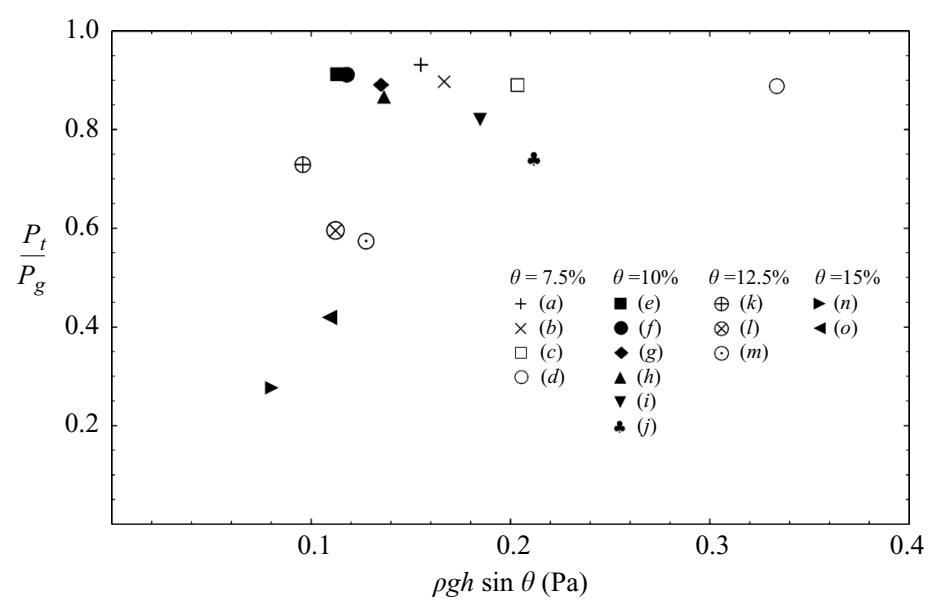

Figure 14. Variation in the ratio $P_{t} / P_{g}$ with the bottom shear stress.

of the solid discharge. This may explain why Bagnold's approach performs poorly at low water discharges for shallow flows on steep slopes.

\subsection{Energy dissipation}

In the absence of particles, energy supplied by gravity is entirely dissipated by turbulent structures. When particles can be entrained, another energy sink is due to momentum and energy transfer between water and particles. To evaluate the efficiency of this energy sink, we compute between the power supplied by gravity to the control volume

$$
P_{g}=\int_{\mathscr{V}} \rho \boldsymbol{g} \cdot \boldsymbol{u} \mathrm{d} \mathscr{V}=\rho g q_{w} L \sin \theta
$$

and the ratio of the turbulent power

$$
P_{t}=\rho g q_{w} L \sin \theta-\bar{N} \bar{F}_{D} \bar{u}_{p} .
$$

In the particle energy budget, we neglect the energy dissipated due to particle spin or lift force. The mean drag force was evaluated using the relation $\bar{F}_{D}=C_{d} \pi a^{2}\left(\bar{u}_{p}-\right.$ $\left.\bar{u}_{f}\right)^{2} / 2$, with $C_{d}=0.5$ the drag coefficient. In the numerical computations, we distinguished the particles in rolling and saltating regimes (see tables 1 and 2).

Figure 14 shows how $P_{t} / P_{g}$ varied with the bottom shear stress $\rho g h \sin \theta$. For gentle slopes $(\tan \theta<0.1)$ and moderate solid discharges $\left(\dot{n}<16\right.$ beads s$\left.^{-1}\right)$, almost all energy was dissipated by turbulence-the ratio $P_{t} / P_{g}$ remains close to $90 \%$-independently of the bottom shear stress. When the solid discharge is increased or for steep slopes, there is a significant drop of the turbulent-energy contribution to the total energy. For instance, for run (n) $\left(\tan \theta=0.15\right.$ and $\dot{n}=16$ beads s$\left.^{-1}\right), 25 \%$ of the energy supplied by gravity is dissipated by turbulence against $75 \%$ by momentum transfers with particles. As for particle velocity, the independence of the ratio $P_{t} / P_{g}$ and $\bar{u}_{f}$ indicated that for our experiments, there was no real coupling between the solid and water phases through energy balance, contrary to what is assumed in Bagnold's approach. 


\section{Concluding remarks}

In recent years, fundamental experiments have been conducted to gain insight into the connection between particle behaviour on the bulk-flow and particle scales to elucidate the dependence of the solid discharge on water flow rate. Typical examples include the experimental and numerical investigations led by Schmeeckle \& Nelson (2003), who tried to clarify the role of turbulence in incipient motion of coarse particles, or the experiments performed by Charru et al. (2004), who analysed entrainment and motion of coarse particles under laminar flow conditions. With the same objective in mind, we ran experiments in a narrow channel, where coarse particles (glass beads) were entrained by a turbulent water stream down a steep slope $(7.5 \%$ to $15 \%)$. As the particle trajectories were two-dimensional, we could measure quantities such as the particle velocity and the number of entrainments/depositions per unit time. Although idealized with respect to sediment transport in mountain streams, our experimental facility provides a realistic basis for understanding the physical processes involved in sediment transport on steep slopes.

The intermittent nature of solid discharge at low water discharges led us to develop a stochastic model that has its roots in earlier work (Einstein 1950), but although this earlier theory was a big step forward it relies on statistical assumptions that have turned out to be unrealistic. For instance, in Einstein's theory and subsequent variants, fluctuations of the solid discharge are Gaussian, whereas field and laboratory measurements show wider fluctuations. This paper proposes a simple theoretical model of events within a control volume. The number of particles within this window can vary as a result of inflow, outflow, entrainment and deposition of particles. Mass balance considerations lead to a birth-death immigration-emigration Markov model, based on the assumption that particles can be entrained both because of water effects and interactions with moving particles. The latter assumption enables feedback between the moving particles and those at rest, and thus helps to explain the existence of wide fluctuations. Indeed, if we remove this feedback $(\mu=0)$, our model provides predictions that closely resemble Einstein's results.

In this purely counting vision of bed-load transport, we do not make the feedback dependent on water flow. Intuitively, we could expect that the water stream cannot entrain an unlimited number of particles because of the energy constraint that limits particle entrainment and transportation. This would favour control of water flow conditions on sediment transport via momentum transfers, as argued by Bagnold $(1966,1973)$ and subsequent authors (e.g. see Seminara et al. 2002). Within this approach, if some part $P_{p}$ of the total energy supplied by acceleration due to gravity is allocated to particle motion, then the total number of moving particles must satisfy

$$
\bar{N}=P_{p} /\left(\bar{F}_{D} \bar{u}_{p}\right),
$$

as shown in $\S 4.6$. Here, it clearly appears experimentally that for bed inclinations as steep as $10 \%$, flow was sufficiently dilute and vigorous for turbulence to be the main energy sink (see figure 14); the energy $P_{p}$ dissipated by particle motion does not exceed $15-20 \%$ of the total and is independent of the fluid velocity. This contradicts Bagnold-like models: if $P_{p}$ remains nearly constant, the number of moving particles should decrease with increasing fluid velocities if $\bar{N}$ is controlled by momentum transfers. With our model, we have shown that the number of moving particles does not depend explicitly on the water flow conditions: to some extent (for $\mu>0$ ), water flow dictates how many particles can be dislodged from the bed and entrained into the flow, but it loosely controls the number of settling particles and the number of 
particles set in motion because of interactions (e.g. collision). In a steady state, the entrainment rate $E=\mu \bar{N}+\lambda_{1}\left(\bar{u}_{f}\right)$ must balance the deposition rate $D=\sigma \bar{N}$, so the total number of moving particles varies nearly linearly with fluid velocity (if $\mu$ and $\sigma$ are constant):

$$
\bar{N}=\frac{\lambda_{1}\left(\bar{u}_{f}\right)}{\sigma-\mu} .
$$

With the solid discharge defined as the product of the number of moving particles $N$ and the particle velocity, our theoretical model was then completed by computing the mean particle velocity. This computation was not indispensable: for our experiments the mean velocity of a particular system did not depend strongly on fluid velocity (see figure 13), whereas for a single particle entrained by the turbulent stream, the particle velocity varies nearly linearly with the mean fluid velocity (Ancey et al. 2002, 2003). A striking and unanticipated result is that the linearity between solid discharge and fluid velocity stems mainly from the dependence of $N$ on $\bar{u}_{f}$.

Our final stochastic model counts the number of moving particles, as depicted by figure 2 . The inflow rate is denoted by $\lambda_{0}$, the outflow rate by $v$, the deposition rate by $\sigma$; for entrainment, we distinguish the entrainment rate induced by water $\left(\lambda_{1}\right)$ from the collective entrainment rate $(\mu \bar{N})$. For flows at equilibrium, the solid discharge $\dot{n}$ corresponds to the inflow and outflow rates: $\dot{n}=\lambda_{0}=\bar{N} \nu$. The other parameters depend on the size of the window and specifically on its length $L$, and the fluid velocity. We cannot compute these parameters analytically, but their dependence on $\bar{u}_{f}$ can be anticipated. Experimentally, we found that $\mu$ and $\sigma$ were almost constant (see figure $12 c$ and table 3 ), whereas $\lambda_{1}$ was nearly proportional to the fluid velocity (see figure $12 b$ ). The model does not distinguish between rolling and saltating particles; a natural development would be to extend it to allow exchanges between them.

This stochastic model enables us to predict a number of macroscopic features of our system. The fluctuations in the solid discharge or the number of moving particles are described well (see figure 7). The model also provides reasonably good estimates of the autocorrelation function and the statistical properties of deposition events for low solid discharges and gentle slopes. At higher solid discharges and for steep slopes, there is an increasing divergence between the model and our data, presumably reflecting the change in behaviour owing to the increase in slope; this change was expected for slopes in excess of $10 \%$ (see $\$ 2.5$ ). Taking a closer look at statistical properties of solid discharge is of fundamental importance to understanding natural phenomena. Here, with our experimental set-up, we concluded that fluctuations arose from interactions between particles and a turbulent stream. This conclusion probably holds true for mountain and gravel-bed rivers, but in that case, local fluctuations may also result from large perturbations of the river on a longer time scale, such as sudden disruption of bedforms, hydraulic instabilities (e.g. local sub-/supercritical transition), and topographic disturbances or changes in the hydrology regime or sediment input (e.g. unstable banks, landsliding mass). There is probably a wide spectrum of time scales corresponding to these sources of fluctuations (from a few years to a few seconds); the fluctuation mechanism we describe in this paper for shallow turbulent flows may be seen as a primary and inherent cause of variability in the sense that it occurs with a short characteristic time dictated by the turbulence time scale, whatever the bed topography and hydraulic conditions (see $\S 2.2$ ).

Simple power-law models relating the sediment flow rate for natural gravel-bed rivers to the water flow depth can perform better than more sophisticated physically based models (Barry et al. 2004). One possible explanation lies in the measurement 
protocols used in the field and laboratory. For gravel-bed rivers, workers in the field of hydraulics and geomorphologists use systems such as the Helley-Smith sampler that measure bed load by capturing sediment over a given time interval. In the laboratory, hydraulics workers impose the solid discharge at the flume inlet (hopper supplying the flume) or measure it at the outlet. For the former, the proper selection of the sampling time (ranging from a few seconds to several minutes) is crucial; this difficulty of selecting a proper time scale is underlined by the large differences among various measurement systems (Bunte \& Abt 2005). In $\$ 2.6$, we saw that the number of settling particles is negative binomial, with mean $r\left(1-p^{\prime}\right) / p^{\prime}$ (see (2.28), where $p^{\prime}$ depends nonlinearly on the time interval $\delta t$ during which the measurement is taken. From this perspective, there is little hope of establishing a direct link between trapped sediment and solid discharge without taking the random nature of transport into account. Experiments in the laboratory pose other problems: since bed load is imposed or measured at the inlet or outlet, the only information available concerns the number of particles that emigrate or immigrate (parameters $v$ and $\lambda_{0}$ of the model) from/into the control volume. At low water discharges, the mean waiting time between two entrainment events is long; if the typical duration of the experiment is shorter than the waiting time, it is likely that no emigrating particle is detected and one may conclude that the solid discharge is zero. From this perspective, the threshold for incipient motion is probably time-dependent at low water discharges, a point never taken into account in hydraulics computations and which may explain the discrepancy between laboratory and field measurements.

\section{REFERENCES}

Aвbott, J. E. \& Francis, J. R. D. 1977 Saltation and suspension trajectories of solid grains in a water stream. Proc. R. Soc. Lond. 284, 225-254.

Abramowitz, M. \& Stegun, I. A. 1964 Handbook of Mathematical Functions. National Bureau of Standards.

Ancey, C., Evesque, P. \& Coussot, P. 1996 Motion of a single bead on a bead row: theoretical investigations. J. Phys. I 6, 725-751.

Ancey, C., Bigillon, F., Frey, P., Lanier, J. \& Ducret, R. 2002 Saltating motion of a bead in a rapid water stream. Phys. Rev. E 66, 036306.

Ancey, C., Bigillon, F., Frey, P. \& Ducret, R. 2003 Rolling motion of a single bead in a rapid shallow water stream down a steep channel. Phys. Rev. E 67, 011303.

Ancey, C., Böhm, T., Jodeau, M. \& Frey, P. 2006 Statistical description of sediment transport experiments. Phys. Rev. E 74, 011302.

Armanini, A. \& Gregoretti, C. 2005 Incipient sediment motion at high slopes in uniform flow condition. Water Resour. Res. 41, W12431.

BAGNOLD, R. A. 1966 An approach to the sediment transport problem from general physics. Professional paper 422-I. United States Geological Survey.

BAgnold, R. A. 1973 The nature of saltation and of 'bed load' transport in water. Proc. R. Soc. Lond. A 332, 473-504.

Balmforth, N. J. \& Provenzale, A. 2001 Patterns of dirt. In Geomorphological Fluid Mechanics (ed. N. J. Balmforth \& A. Provenzale), pp. 369-393. Springer.

Barry, J. J., Buffington, J. M. \& King, J. G. 2004 A general power equation for predicting bed load transport rates in gravel bed rivers. Water Resour. Res. 40, W10401.

Böhm, T., Ancey, C., Frey, P., Reboud, J.-L. \& Duccotet, C. 2004 Fluctuations of the solid discharge of gravity-driven particle flows in a turbulent stream. Phys. Rev. E 69, 061307.

Böhm, T., Frey, P., Duccotet, C., Ancey, C., Jodeau, M. \& Reboud, J.-L. 2006 Two-dimensional motion of a set of particles in a free surface flow with image processing. Exps. Fluids 41, $1-11$. 
Bunte, K. \& Aвт, S. 2005 Effect of sampling time on measured gravel bed load transport rates in a coarse-bedded stream. Water Resour. Res. 41, W11405.

Charru, F., Mouilleron, H. \& Eiff, O. 2004 Erosion and deposition of particles on a bed sheared by a viscous flow. J. Fluid Mech. 519, 55-80.

Cheng, N.-S. 2004 Analysis of bedload transport in laminar flows. Adv. Water Resour. 27, 937-942.

Cheng, N.-S., Tang, H. \& Zhu, L. 2004 Evaluation of bed load transport subject ot high shear stress fluctuations. Water Resour. Res. 40, W05601.

Cox, D. R. \& Miller, H. D. 1965 The Theory of Stochastic Processes. Chapman \& Hall.

Drake, T. G., Shreve, R. L., Dietrich, W. E. \& Leopold, L. B. 1988 Bedload transport of fine gravel observed by motion-picture photography. J. Fluid Mech. 192, 193-217.

Einstern, H. 1950 The bed-load function for sediment transportation in open channel flows. Tech. Rep. 1026. United States Department of Agriculture.

Ettema, R. \& Mutel, C. 2004 Hans Albert Einstein: Innovation and compromise in formulating sediment transport by rivers. J. Hydraul. Engng ASCE 130, 477-487.

FERNANDEZ LuQUe, R. \& VAN BEEK, R. 1976 Erosion and transport of bed-load sediment. J. Hydraul. Res. 14, 127-144.

Frey, P. \& ReBoud, J.-L. 2001 Experimental study of narrow free-surface turbulent flows on steep slopes. In Advances in flow modeling and turbulence measurements (ed. H. Ninokata, A. Wada \& N. Tanaka). World Scientific.

Frey, P., Dufresne, M., Böhm, T., Jodeau, M. \& Ancey, C. 2006 Experimental study of bed load on steep slopes. In River Flow 2006 (ed. R. M. L. Ferreira, E. C. T. L. Alves, J. G. A. B. Leal \& A. H. Cardoso), vol. 1, pp. 887-893. Taylor \& Francis.

Gardiner, C. W. 1983 Handbook of Stochastic Methods. Springer.

Gomez, B. \& CHurch, M. 1989 An assessment of bed load sediment transport formulae for gravel bed rivers. Water Resour. Res. 25, 1161-1186.

Gordon, R., CARMichael, J. B. \& Isackson, F. J. 1972 Saltation of plastic balls in a one-dimensional flume. Water Resour. Res. 8, 444-459.

GraF, W. H. 1984 Hydraulics of Sediment Transport. Littleton.

Grant, G. E. 1997 Critical flow constrains flow hydraulics in mobile-bed streams: a new hypothesis. Water Resour. Res. 33, 349-358.

IVERSON, R. M. 2003 How should mathematical models of geomorphic processes be judged? In Prediction in Geomorphology (ed. P. R. Wilcock \& R. M. Iverson), pp. 83-94. American Geophysical Union.

Jenkins, J. T. \& Hanes, H. M. 1998 Collisional sheet flows of sediment driven by a turbulent fluid. J. Fluid Mech. 370, 29-52.

Julien, P.-Y. 1994 Erosion and Sedimentation. Cambridge University Press.

Kirchner, J., Dietrich, W., Iseya, F. \& IKedA, H. 1990 The variability of critical shear stress, friction angle, and grain protrusion in water-worked sediments. Sedimentology 37, 647-672.

Kleinhans, M. \& van RiJn, L. 2002 Stochastic prediction of sediment transport in sand-gravel bed rivers. J. Hydraul. Engng ASCE 128, 412-425.

Kovacs, A. \& Parker, G. 1994 A new vectorial bedload formulation and its application to the time evolution of straight rivers. J. Fluid Mech. 267, 153-183.

Kuhnle, R. A. \& Southard, J. B. 1988 Bed load transport fluctuations in a gravel bed laboratory channel. Water Resour. Res. 24, 247-260.

Laursen, E. M. 1999 Discussion on 'Pickup probability for sediment entrainment'. J. Hydraul. Engng ASCE 124, 786-787.

Lisle, I. G., Rose, C. W., Hogarth, W. L., Hairsine, P. B., Sander, G. \& Parlange, J. Y. 1998 Stochastic sediment transport in soil erosion. J. Hydrol. 204, 217-230.

LisLE, T. E. 1989 Sediment transport and resulting deposition in spawning gravels, north coastal California. Water Resour. Res. 25, 1303-1319.

Lopez, F. \& Garcìa, M. 2001 Risk of sediment erosion and suspension in turbulent flows. J. Hydraul. Engng ASCE 127, 231-235.

Martin, Y. 2003 Evaluation of bed load transport formulae using field evidence from the Vedder River, British Columbia. Geomorphology 53, 75-95.

Meyer-Peter, E. \& Müller, R. 1948 Formulas for bed load transport. In 2nd meeting (ed. IAHR), Stockholm, Sweden, pp. 39-64. 
Nelson, J. M., Shreve, R. L., Mclean, S. R. \& Drake, T. G. 1995 Role of near-bed turbulence structure in bed load transport and bed form mechanics. Water Resour. Res. 31, 2071-2086.

Nelson, J. M., Schmeeckle, M. W. \& Shreve, R. L. 2001 Turbulence and particle entrainment. In Gravel Bed Rivers (ed. M. Mosley), pp. 221-248. Littleton.

NeZU, I. \& Nakagawa, H. 1993 Turbulence in Open-Channel Flows. Balkema.

NiÑo, Y. \& GARcìA, M. H. 1994 Gravel saltation 2. Modeling. Water Resour. Res. 30, 1915-1924.

NiÑo, Y. \& GARCìA, M. H. 1998 Using Lagrangian particle saltation observations for bedload sediment transport modelling. Hydrolog. Process. 12, 1197-1218.

Niño, Y., Garcìa, M. H. \& Ayala, L. 1994 Gravel saltation 1. Experiments. Water Resour. Res. 30, 1907-1914.

Nikora, V., Heald, J., Goring, D. \& McEwan, I. 2001 Diffusion of saltating particles in unidirectional water flow over a rough granular bed. J. Phys. A: Math. Gen. 34, L743-L749.

Nikora, V., Habersack, H., Huber, T. \& McEwan, I. 2002 On bed particle diffusion in gravel bed flows under weak bed load transport. Water Resour. Res. 38, WR000513.

Paintal, A. 1971 A stochastic model of bed load transport. J. Hydraul. Res. 9, 527-554.

Papanicolaou, A., Diplas, P., Evaggelopoulos, N. \& Fotopoulos, S. 2002 Stochastic incipient motion criterion for spheres under various bed packing conditions. J. Hydraul. Engng ASCE 128, 369-390.

RaudKIVI, A. 1990 Loose Boundary Hydraulics. Pergamon.

Rickenmann, D. 2001 Comparison of bed load transport in torrents and gravel bed streams. Water Resour. Res. 37, 3295-3306.

VAN RIJN, L. 1985 Sediment transport, part I: bed load transport. J. Hydraul. Engng ASCE 110, $1431-1456$.

Schmeeckle, M. W. \& Nelson, J. M. 2003 Direct numerical simulation of bedload transport using a local, dynamic boundary condition. Sedimentology 50, 279-301.

Schmeeckle, M. W., Nelson, J. M., Pitlick, J. \& Bennett, J. 2001 Interparticle collision of natural sediment grains in water. Water Resour. Res. 37, 2377-2391.

Schmeeckle, M., Nelson, J. M. \& Shreve, R. 2007 Forces on stationary particles in near-bed turbulent flows. J. Geophys. Res. 112, F02003.

Seminara, G., Solari, L. \& Parker, G. 2002 Bed load at low Shield stress on arbitrarilty sloping beds: failure of the Bagnold hypothesis. Water Resour. Res. 38, 1249.

Smart, G. \& JAEGGI, M. 1983 Sedimenttransport in steilen Gerinnen. Tech. Rep. 64. Mitteilungen der Versuchanstalt für wasserbau, Hydrologie und Glaziologie.

Sornette, D. 2000 Critical Phenomena in Natural Sciences. Springer.

Wiberg, P. L. \& Smith, J. D. 1985 A theoretical model for saltating grains in water. J. Geophys. Res. C 90, 7341-7354.

WiberG, P. L. \& Smith, J. D. 1989 Model for calculating bedload transport of sediment. J. Hydraul. Engng ASCE 115, 101-123.

WILCOCK, P. R. 2001 Toward a practical method for estimating sediment-transport rates in gravel bed-rivers. Earth Surf. Processes Landforms 26, 1395-1408.

Wong, M. \& PARKer, G. 2006 Reanalysis and correction of bed-load relation of Meyer-Peter and Müller using their own database. J. Hydraul. Engng ASCE 132, 1159-1168. 